\title{
Differences in Metformin and Thiamine Uptake between Human and Mouse Organic Cation Transporter 1: Structural Determinants and Potential Consequences for Intrahepatic Concentrations ${ }^{\mathbb{\$}}$
}

\author{
Marleen J. Meyer, Alzbeta Tuerkova, Sarah Römer, Christoph Wenzel, Tina Seitz, \\ Jochen Gaedcke, Stefan Oswald, Jürgen Brockmöller, (1)Barbara Zdrazil, \\ and (1) Mladen V. Tzvetkov
}

Institute of Pharmacology, Center of Drug Absorption and Transport (C_DAT), University Medicine Greifswald, Greifswald, Germany (M.J.M., S.R., C.W., S.O., M.V.T.); Department of Pharmaceutical Chemistry, Division of Drug Design and Medicinal Chemistry, University of Vienna, Vienna, Austria (A.T., B.Z.); and Department of General, Visceral, and Pediatric Surgery (J.G.) and Institute of Clinical Pharmacology (T.S., J.B.), University Medical Center Göttingen, Göttingen, Germany

Received July 6, 2020; accepted September 28, 2020

\section{ABSTRACT}

The most commonly used oral antidiabetic drug, metformin, is a substrate of the hepatic uptake transporter OCT1 (gene name SLC22A1). However, OCT1 deficiency leads to more pronounced reductions of metformin concentrations in mouse than in human liver. Similarly, the effects of OCT1 deficiency on the pharmacokinetics of thiamine were reported to differ between human and mouse. Here, we compared the uptake characteristics of metformin and thiamine between human and mouse OCT1 using stably transfected human embryonic kidney 293 cells. The affinity for metformin was 4.9 -fold lower in human than in mouse OCT1, resulting in a 6.5-fold lower intrinsic clearance. Therefore, the estimated liver-to-blood partition coefficient is only 3.34 in human compared with 14.4 in mouse and may contribute to higher intrahepatic concentrations in mice. Similarly, the affinity for thiamine was $\mathbf{9 . 5}$-fold lower in human than in mouse OCT1. Using human-mouse chimeric OCT1, we showed that simultaneous substitution of transmembrane helices TMH2 and TMH3 resulted in the reversal of affinity for metformin. Using homology modeling, we suggest several explanations, of which a different interaction of Leu155 (human
TMH2) compared with Val156 (mouse TMH2) with residues in TMH3 had the strongest experimental support. In conclusion, the contribution of human OCT1 to the cellular uptake of thiamine and especially of metformin may be much lower than that of mouse OCT1. This may lead to an overestimation of the effects of OCT1 on hepatic concentrations in humans when using mouse as a model. In addition, comparative analyses of human and mouse orthologs may help reveal mechanisms of OCT1 transport.

\section{SIGNIFICANCE STATEMENT}

OCT1 is a major hepatic uptake transporter of metformin and thiamine, but this study reports strong differences in the affinity for both compounds between human and mouse OCT1. Consequently, intrahepatic metformin concentrations could be much higher in mice than in humans, impacting metformin actions and representing a strong limitation of using rodent animal models for predictions of OCT1-related pharmacokinetics and efficacy in humans. Furthermore, OCT1 transmembrane helices TMH2 and TMH3 were identified to confer the observed species-specific differences in metformin affinity.

\section{Introduction}

Metformin is the most commonly prescribed oral antidiabetic drug. It reduces plasma glucose and has favorable effects on lipid metabolism.

This work was supported in part by European Regional Development Fund (ERDF) [Grant GHS-18-0021] to M.V.T.

Part of this work has been presented in the poster "OCT1 of mice and men species-specific differences in the function of OCT1" by Meyer, MJ; Bolesta, M; Schreier, P; Krätzner, R; Seitz, T; Brockmöller, J; Tzvetkov, MV at the 11th International BioMedical Transporters Conference, Aug. 4-8, 2019 in Lucerne, Switzerland. This work is part of the Ph.D. thesis of M.J.M.

https://doi.org/10.1124/dmd.120.000170.

SThis article has supplemental material available at dmd.aspetjournals.org.
Metformin acts both in the liver and in the gut (Rena et al., 2017). In hepatocytes, metformin decreases glucose production and lipogenesis both by AMPK-dependent and AMPK-independent mechanisms.

At physiologic $\mathrm{pH}$, metformin is almost entirely present as organic cation $\left(\mathrm{pK}_{\mathrm{a}}\right.$ of 12.04 , estimated $99.998 \%$ positively charged molecules). Therefore, metformin depends highly on transporter proteins to enter hepatocytes. The organic cation transporter 1 (OCT1, gene name $S L C 22 A 1)$, which is strongly expressed in the sinusoidal membrane of hepatocytes, has been demonstrated to be the major hepatic uptake transporter of metformin (Wang et al., 2002; Shu et al., 2007). In humans, OCT1 is genetically highly variable. In total, $9 \%$ of Europeans and white Americans are carriers of two reduced function or loss-of-function OCT1 alleles and are so-called poor OCT1

ABBREVIATIONS: AMPK, AMP-activated protein kinase; DPBS, Dulbecco's phosphate-buffered saline; GLUT3, glucose 3 transporter; h, human; HBSS, Hanks' buffered salt solution; HEK, human embryonic kidney; IS, internal standard; IVIVE, in vitro to in vivo extrapolation; $\mathrm{K}_{\mathrm{p}}$, liver-to-blood partition coefficient; LC-MS/MS, liquid chromatography tandem mass spectrometry; m, mouse; MFS, major facilitator superfamily; OCT, organic cation transporter; TMH, transmembrane helix PET, positron emission tomography; DAPI, 4',6-diamidino-2-phenylindole; THTR, thiamine transporter; SLC, solute carrier. 
transporters (Kerb et al., 2002; Shu et al., 2003; Tzvetkov et al., 2012; Seitz et al., 2015). In some specific populations, like Surui Indians, this percentage may increase up to $80 \%$ (Seitz et al., 2015). These genetic OCT1 variants are expected to affect the hepatic uptake and thus the efficacy of metformin.

Indeed, OCT1 knockout in mice reduced metformin concentrations in the liver by up to 30-fold (Wang et al., 2002, 2003) and abolished the glucose-lowering effects of metformin (Shu et al., 2007). OCT1 deficiency in mice has been suggested to lead to an absolute lack of metformin uptake into hepatocytes (Wang et al., 2002). Also in humans, genetic variants leading to decreased OCT1 activity were associated with reduced intrahepatic concentrations of metformin (Sundelin et al., 2017). However, despite some initial reports of reduced response to metformin in poor OCT1 transporters (Shu et al., 2007), larger studies and meta-analyses could not confirm the association of OCT1 genetic variants with reduced efficacy of metformin in humans (Zhou et al., 2009; Dujic et al., 2017).

Similarly, OCT1 was identified as a relevant transporter of thiamine (vitamin B1) in the mouse liver (Chen et al., 2014). OCT1 knockout in mice resulted in higher thiamine plasma levels, likely because of decreased hepatic extraction (Chen et al., 2014; Liang et al., 2018). However, a recent study in humans showed no differences in the plasma concentrations of thiamine or its metabolites in poor OCT1 transporters (Jensen et al., 2020). Taken together, this questions the suitability of mouse as a model for studying effects of OCT1 on metformin or thiamine-driven biologic processes without understanding the causes of the species differences.

One possible explanation for the variable results between human and mouse may be differences in the kinetics of OCT1-mediated uptake of metformin and thiamine. Although species differences in organ-specific OCT1 expression are well characterized (Gorboulev et al., 1997; Zhang et al., 1997; Green et al., 1999; Schmitt et al., 2003), there is only very limited data reporting differences in transport activity. The amino acid identity between the human and mouse OCT1 orthologs is $77 \%$. Since the exact mechanism of substrate interaction with OCT1 is not known, it is difficult to predict to what extent the $23 \%$ different amino acids between the two orthologs can confer differences in uptake. Furthermore, metformin and thiamine were suggested to share common sites of ligand-transporter interaction within OCT1 (Chen et al., 2014). Therefore, similar differences in the uptake between human and mouse could be expected for these two substrates.

The aim of this study was to compare the uptake of metformin and thiamine by human and mouse OCT1 in vitro to explore underlying mechanisms causing differences in the hepatic concentrations in humans and mice. This should help to better interpret the data of mouse models and should improve the translation of mouse pharmacokinetic data to humans. Furthermore, we used the differences in uptake between human and mouse OCT1 as a tool to improve our understanding of the transport mechanism of OCT1.

\section{Materials and Methods}

Reagents. Metformin hydrochloride, thiamine hydrochloride, ammoniumbicarbonate, dithiothreitol, and iodoacetamide were obtained from Sigma-Aldrich (Taufkirchen, Germany); buformin hydrochloride was obtained from Wako Chemicals (Neuss, Germany); and thiamine-d3 hydrochloride was obtained from Toronto Research Chemicals (North York, ON, Canada). All chemicals used in this study were purchased from commercial sources and had purities of $95 \%$ or higher. Dulbecco's modified Eagle's medium (DMEM), Hanks' buffered salt solution (HBSS), and additives for cell culturing were obtained from Life Technologies (Darmstadt, Germany). Dulbecco's phosphate-buffered saline (DPBS) was obtained from PAN-Biotech (Aidenbach, Germany). Poly-D-lysine (1-5 kDa), HEPES, bicinchoninic acid, and copper sulfate pentahydrate were obtained from Sigma-Aldrich. Twelve-well plates were obtained from Nunc (Langenselbold, Germany), and tissue culture flasks were from Sarstedt (Nümbrecht, Germany). Acetonitrile, methanol, and formic acid in LC-MS/MS grade and sodium chloride were obtained from Merck (Darmstadt, Germany). SDS (ultrapure) was obtained from AppliChem (Darmstadt, Germany). Sequencing Grade Modified Trypsin and ProteaseMAX surfactant were obtained from Promega (Mannheim, Germany).

Generation of OCT1 Constructs. For overexpression of OCT1 in human embryonic kidney (HEK) 293 cells, pcDNA5/FRT expression vectors (Thermo Fisher Scientific, Darmstadt, Germany) containing wild-type, mutant, or chimeric OCT1 constructs were generated as follows or as described previously (Tzvetkov et al., 2012; Seitz et al., 2015). Human-mouse chimeric OCT1 was generated by restriction of human and mouse OCT1 genes with Bsu36I and BsaBI, separating the OCT1 gene into three fragments: from $\mathrm{N}$ terminus to large intracellular loop, from transmembrane helix (TMH) 7 to TMH9, and from TMH10 to C terminus (Fig. 3A). The fragments were ligated back together in the correct order but with different combinations of the species, and the resulting chimeric OCT1 genes were cloned into the pcDNA5/FRT vector after restriction of both gene and vector with HindIII and EcoRV. These constructs were then used for targeted chromosomal integration into HEK293 cells. Human-mouse chimeric OCT1 constructs with single TMH substitutions were generated using the overlap extension method (Horton et al., 1989) and primers listed in Supplemental Table 1. Point mutations in human and mouse OCT1 genes were introduced by site-directed mutagenesis in pcDNA5/FRT vectors containing human or mouse OCT1 wild-type genes, using primers listed in Supplemental Table 1. All generated constructs were validated by capillary sequencing of the complete open reading frame of OCT1 before transfection into HEK293 cells.

Cell Lines and Cell Culturing. HEK293 cells stably overexpressing human OCT1, mouse OCT1, rat OCT1, human-mouse chimeric OCT1, or human OCT2 were generated by targeted chromosomal integration using the Flp-In System (Life Technologies) as described previously (Tzvetkov et al., 2012; Seitz et al., 2015). Cells were cultured in Dulbecco's modified Eagle's medium supplemented with $10 \%$ FBS, $100 \mathrm{U} / \mathrm{ml}$ penicillin, and $100 \mu \mathrm{g} / \mathrm{ml}$ streptomycin at $37^{\circ} \mathrm{C}$ and $5 \% \mathrm{CO}_{2}$.

Transient Transfection of T-REx-293 Cells for Cellular Uptake Experiments. For transient transfection of OCT1 constructs into HEK293 cells for uptake experiments, $5 \times 10^{5}$ T-REx-293 cells (Life Technologies) were seeded per well of a 12-well plate precoated with poly-D-lysine. At 24 hours later, the cells were transfected with $100 \mu \mathrm{l}$ of reaction mix per well, containing $2 \mu \mathrm{g}$ pcDNA5/ FRT vector with the OCT1 construct of interest, $0.5 \mu \mathrm{g}$ pGFP-tpz vector, and 6.25 $\mu l$ Lipofectamine 2000 (Thermo Fisher Scientific), according to the manufacturer's instructions. At 48 hours later, transfection efficacy was assessed microscopically by visualizing the GFP signal of the cotransfected GFP vector, and the cells were used for uptake experiments.

Cellular Uptake Experiments. At 48 hours prior to the experiment, $6 \times 10^{5}$ cells were seeded per well of a 12 -well plate. When using transiently transfected cells, $5 \times 10^{5}$ T-REx-293 cells were seeded per well of a 12-well plate 72 hours prior to the experiment, and they were transfected 24 hours later as described above. Twelve-well plates were precoated with poly-D-lysine.

Cellular uptake experiments were performed at $37^{\circ} \mathrm{C}$ and $\mathrm{pH} 7.4$ using HBSS supplemented with $10 \mathrm{mM}$ HEPES (in the following referred to as HBSS+). Cells were washed once with $1 \mathrm{ml}$ of prewarmed $\left(37^{\circ} \mathrm{C}\right) \mathrm{HBSS}+$, and uptake was started by adding $400 \mu \mathrm{l}$ prewarmed HBSS+ containing the substrate. Uptake was stopped after 2 minutes by adding $2 \mathrm{ml}$ ice-cold HBSS+. Cells were washed twice with $2 \mathrm{ml}$ ice-cold HBSS+ and were lysed in $500 \mu \mathrm{l} 80 \%$ acetonitrile supplemented with internal standard (Table 1). Intracellular substrate concentrations were measured by LC-MS/MS as described below and afterward were normalized to the total amount of protein in the sample as measured using the bicinchoninic acid assay (Smith et al., 1985).

Quantification of Intracellular Substrate Concentration by LC-MS/MS. For quantification of intracellular substrate concentrations, the cell debris was removed by centrifugation of the cell lysate at $16,000 \mathrm{~g}$ for 15 minutes. In total, $350 \mu 1$ of the supernatant was evaporated to dryness under nitrogen flow at $40^{\circ} \mathrm{C}$. The sample was reconstituted in $200 \mu \mathrm{l} 0.1 \%$ formic acid, and 5 or $15 \mu \mathrm{l}$ was injected into the LC-MS/MS system for metformin and thiamine, respectively.

For LC-MS/MS quantification, an API 4000 tandem mass spectrometer (AB SCIEX, Darmstadt, Germany) was used. Samples were separated on a Brownlee SPP RP-Amide column $(4.6 \times 100 \mathrm{~mm}, 2.7 \mu \mathrm{m}$; PerkinElmer, Rodgau, 
TABLE 1

Parameters of quantitative LC-MS/MS analyses

\begin{tabular}{|c|c|c|c|c|c|c|c|}
\hline Analyte & Quantifier Precursor Ion to Product Ion $(\mathrm{m} / \mathrm{z})$ & $\begin{array}{l}\text { Retention } \\
\text { Time (min) }\end{array}$ & IS & IS Precursor Ion to Product Ion (m/z) & $\begin{array}{l}\text { Retention Time } \\
\text { IS (min) }\end{array}$ & $\begin{array}{c}\text { Mobile Phase } \\
{\text { (\% Organic Solvent })^{a}}\end{array}$ & $\begin{array}{l}\text { Flow } \\
(\mu \mathrm{l} / \mathrm{min})\end{array}$ \\
\hline Metformin & $130.1>71$ & 2.88 & Buformin & $158.1>60$ & 4.0 & 3 & 300 \\
\hline Thiamine & $265.3>122$ & 2.47 & Thiamine-d3 & $269.1>125$ & 2.47 & 3 & 350 \\
\hline
\end{tabular}

$\mathrm{m} / \mathrm{z}$, mass-to-charge ratio.

${ }^{a}$ Six parts acetonitrile + one part methanol.

Germany) using a mobile phase of $0.1 \%$ (v/v) formic acid and varying concentrations of organic solvent (parameters are listed in Table 1).

Immunocytochemical Staining and Confocal Microscopy Analysis of OCT1-Overexpressing Cells. For immunocytochemical staining of OCT1, $6 \times$ $10^{5}$ HEK293 cells stably overexpressing human, mouse, or human-mouse chimeric OCT1 were seeded onto coverslips in 12-well plates 48 hours prior to the experiment. Coverslips were precoated with poly-D-lysine. Cells were washed twice with $1 \mathrm{ml}$ DPBS for 10 minutes and were fixed with $100 \%$ ethanol for 20 minutes at $-20^{\circ} \mathrm{C}$. After washing three times with DPBS for 5 minutes, cell membranes were permeabilized with DPBS/ $0.4 \%$ Tween 20 for 10 minutes. Cells were washed three times with DPBS for 5 minutes and blocked with blocking buffer (DPBS/5\% FBS) for 1-3 hours. Cells were incubated with the primary antibodies diluted in blocking buffer (according to Supplemental Table 2) in a humid chamber overnight. The next day, after washing three times with DPBS for 5 minutes, the cells were incubated with the secondary antibodies diluted in blocking buffer (according to Supplemental Table 2) for 1 to 2 hours protected from light. After washing three times with DPBS for 5 minutes, coverslips were mounted with ROTI-Mount FluorCare DAPI (Carl Roth, Karlsruhe, Germany) onto microscope slides. The cells were analyzed using a laser scanning microscope (LSM780; Carl Zeiss, Jena, Germany), and the images were processed using the Fiji distribution of ImageJ2 (Schindelin et al., 2012; Rueden et al., 2017).

Quantification of OCT1 Protein Abundance by Targeted Proteomics. Normal human liver tissue was obtained as excess material, which had to be removed for technical reasons during liver surgery. Patients had given their informed consent for research use of the tissues, and the procedures were approved by the ethics committee of the University Medicine Göttingen, Georg-August-Universität Göttingen (application number 26/01/17). Preparation of murine liver was carried out in compliance with the German laws on animal welfare ( $₫ 4$ Absatz 3 TierSchG), and all animals used were reported to the Landesveterinär- und Lebensmitteluntersuchungsamt Mecklenburg-Vorpommern.

Human $(N=12$, eight female and four male $)$ and murine $[N=18$, each $N=$ 6 of C57BL/6N (two female and four male), C57BL/6J, and FVB mice (each three female and three male)] liver samples were mechanically crushed in a stainless-steel mortar system, precooled in liquid nitrogen. Approximately $100 \mathrm{mg}$ tissue powder was used for isolation of native integral membrane using the ProteoExtract Native Membrane Extraction Kit according to the manufacturer's instructions (Merck). Tissues were additionally homogenized in a glass douncer during the step of cell lysis. Cell pellets of OCT1overexpressing cells were directly used for extraction. Total protein content of the resulting membrane fraction was determined by bicinchoninic acid assay. If necessary, membrane fractions were adjusted to a maximum protein amount of $2 \mu \mathrm{g} / \mu \mathrm{l}$. Subsequently, $100 \mu \mathrm{l}$ of each membrane fraction was mixed with $10 \mu \mathrm{l}$ dithiothreitol $(200 \mathrm{mM}), 40 \mu \mathrm{l}$ ammonium bicarbonate buffer $(50 \mathrm{mM}, \mathrm{pH} 7.8)$, and $10 \mu$ l ProteaseMAX $(1 \%, \mathrm{~m} / \mathrm{v})$ and incubated for 30 minutes at $60^{\circ} \mathrm{C}$ (denaturation). After cooling down, $10 \mu \mathrm{l}$ iodoacetamide $(400 \mathrm{mM})$ was added, and the samples were incubated in a darkened water bath for 15 minutes at $37^{\circ} \mathrm{C}$ (alkylation). For protein digestion, $10 \mu \mathrm{l}$ trypsin (trypsin/protein ratio of $1: 40$ ) was added, and samples were incubated in a water bath for 16 hours at $37^{\circ} \mathrm{C}$. Digestion was stopped by addition of $20 \mu \mathrm{l}$ formic acid $(10 \%, \mathrm{v} / \mathrm{v})$. All samples were stored at $-80^{\circ} \mathrm{C}$ until further processing. Finally, $35 \mu$ of the digested membrane fraction was mixed with $35 \mu$ l isotope-labeled internal standard (IS) peptide mix (10 $\mathrm{nM}$ of each IS; Thermo Fisher Scientific). All sample preparation and digestion steps were performed using Protein LoBind tubes (Eppendorf, Hamburg, Germany). Protein quantification was conducted on a 5500
QTRAP triple quadrupole mass spectrometer (AB Sciex) coupled to an Agilent Technologies 1260 Infinity system (Agilent Technologies) using validated LC-MS/MS methods as recently described (Drozdzik et al., 2019). Transporter proteins and the respective proteospecific peptides and the stable isotope-labeled internal standard peptides considered in our analysis are given in Supplementary Table 3. Protein abundance of human OCT1 was determined by using three peptides, whereas mouse OCT1 and $\mathrm{Na}^{+} / \mathrm{K}^{+}$ATPase were determined by using one peptide. For each peptide, two to three mass transitions have been monitored.

IVIVE to Estimate the Liver Partition Coefficient of Metformin. The uptake of metformin across the sinusoidal membrane into the liver was estimated based on in vitro uptake measurements in stably transfected HEK293 cells overexpressing human or mouse OCT1. In vitro clearance $\left(C L_{\text {in }}\right.$ vitro $)$ was calculated as follows:

$$
C L_{\text {in vitro }}=\frac{v_{\max }}{K_{M}},
$$

where $v_{\max }$ is the maximum transport rate (picomole $\times$ minute $^{-1} \times$ milligram protein $^{-1}$ ) and $\mathrm{K}_{\mathrm{M}}$ is the Michaelis constant (micromolar) determined in HEK293 cells. The obtained $C L_{\text {in vitro }}\left(\right.$ microliter $\times$ minut $^{-1} \times$ milligram protein $\left.^{-1}\right)$ was used for extrapolation toward total human or mouse liver clearance, which was mediated by active transport via human or mouse OCT1, respectively.

The active OCT1-mediated uptake into the liver $\left(C L_{i n, a c t}\right)$ was calculated as follows:

$$
C L_{\text {in }, a c t}=C L_{\text {in vitro }} \times \frac{E_{\text {in vivo }}}{E_{\text {in vitro }}} \times L W \times \text { total protein per unit } L W,
$$

where $E$ refers to the total OCT1 expression in human or mouse liver $\left(E_{\text {in vivo }}\right)$ and in HEK293 cells overexpressing human or mouse OCT1 $\left(E_{\text {in vitro }}\right)$ (picomole $\times$ milligram protein ${ }^{-1}$ ). In this case, we assumed that $100 \%$ of the OCT1 protein is localized in the plasma membrane both in the liver and in HEK293 cells. $L W$ refers to the liver weight in human and mouse (gram), respectively. The total protein amount per unit $L W$ is given as (milligram protein $\times$ gram liver $^{-1}$ ) and was obtained from Sohlenius-Sternbeck (2006).

Passive diffusion into the liver $\left[C L_{\text {diff }}\right.$ (microliter $\times$ minute $^{-1} \times$ milligram protein $^{-1}$ )] was estimated by using HEK293 cells transfected with the empty pcDNA5/FRT vector and was calculated as follows:

$$
C L_{\text {diff }}=C L_{\text {in vitro }} \times L W \times \text { total protein per unit } L W
$$

The liver partition coefficient $\left(K_{p}\right)$ was calculated based on the extended clearance concept according to Guo et al. (2018):

$$
K_{p}=\frac{C L_{i n, a c t}+C L_{i n, \text { diff }}}{C L_{e f, a c t}+C L_{e f, d i f f}+C L_{\text {bile }}+C L_{m e t}}
$$

The following simplifications were made based on the findings that metformin is neither metabolized nor significantly excreted by transporters or secreted into the bile (Pentikäinen et al., 1979; Tucker et al., 1981): the clearances for transportermediated efflux $\left(C L_{\text {ef.act }}\right)$, for biliary excretion of unchanged drug $\left(C L_{\text {bile }}\right)$, and for metabolism $\left(C L_{m e t}\right)$ were set to zero. Furthermore, we assumed that the passive influx diffusion permeation $\left(C L_{i n}\right.$, diff $)$ is equal to the passive efflux diffusion permeation $\left(C L_{e f, \text { diff }}\right)$, here further designated simply as $C L_{\text {diff. }} K_{p}$ was predicted using the following equation (Yabe et al., 2011; Shitara et al., 2013):

$$
K_{p}=\frac{C L_{i n, a c t}+C L_{d i f f}}{C L_{d i f f}}
$$


As metformin had been shown to have negligible protein binding, the fraction unbound in plasma can be assumed as 1 (Tucker et al., 1981). The prediction of the liver partition coefficient for unbound drug concentration thereby was assumed as

$$
K_{p, u}=K_{p}
$$

Computational Modeling. Available templates for structural modeling were identified by using fold recognition methods offered by the pGenThreader server (available at http://bioinf.cs.ucl.ac.uk/psipred/) (Lobley et al., 2009). The human glucose 3 transporter (GLUT3; Protein Data Bank identifier (PDB ID) 4zw9) was selected as an optimal template for both human and mouse OCT1 modeling (Deng et al., 2015). This crystal structure of GLUT3 was selected based on multiple criteria, such as being resolved in high resolution (1.5 $⿱$ ) and adopting an outward-occluded conformation, which is particularly useful for the investigation of substrate binding. Sequence-to-structure alignment between GLUT3 and human and mouse OCT1 sequence, respectively, was initially generated in PROMALS3D and subsequently revised and corrected by manual intervention (Pei et al., 2008). The large extracellular loop between TMH1 and TMH2 (88 residues) and one C-terminal intracellular loop (22 residues) were lacking structural templates and were therefore omitted for structural modeling purposes.

In total, 100 structural models were generated for both human and mouse OCT1 using Modeler 9.17 (Eswar et al., 2006). Energy minimization was performed to optimize the orientation of side chains. AMBER99SB-ILDN force field (Lindorff-Larsen et al., 2010) and GROMACS version 5.1.4 (Abraham et al., 2015) were used for steepest descent minimization. The convergence criterion was set to a maximum force $<100.0 \mathrm{~kJ} / \mathrm{mol}$ per nanometer. The final models for human and mouse OCT1 were selected on the basis of the MolProbity score ranking (http://molprobity.biochem.duke.edu/) and a proper orientation of the Asp474/475 residue, which is the main residue known to be implicated in ligand binding. Ramachandran outliers were visually inspected in Molecular Operating Environment 19 (Chemical Computing Group ULC, Montreal, QC, Canada). In silico models generated for human OCT1 and mouse OCT1 are available as supplement to this publication (Supplemental Files hOCT1.pdb and mOCT1.pdb).

Two independent algorithms-FTSite (Ngan et al., 2012) and SiteFinder in Molecular Operating Environment 19-were used to identify possible interaction sites in human and mouse OCT1 transporters. The FTSite program docks 16 small probe molecules to identify hot spots in the protein structure. Probe molecules are clustered, and the poses are ranked according to the empirical free energy function. The SiteFinder tool in MOE utilizes the alpha sphere method in which the protein cavities are explored by virtual spheres generated in the site. Every sphere can also differentiate with respect to potential hydrophobic or hydrophilic contacts. Predicted binding sites are ranked according to the number of alpha spheres located in every detected binding site. Standard settings of SiteFinder were applied. Hydrophobic interactions were analyzed by a helical wheel projection using DrawCoil 1.0 (available at https://grigoryanlab.org/drawcoil/).

Data Analyses. Kinetic parameters of metformin and thiamine transport $\left(\mathrm{K}_{\mathrm{M}}\right.$ and $\mathrm{v}_{\max }$ ) were determined by nonlinear regression to the Michaelis-Menten equation using GraphPad Prism version 5.01 (GraphPad Software Inc., La Jolla, $\mathrm{CA})$. The kinetic parameters or uptake values were compared between human and mouse OCT1, human-mouse chimeric OCT1, or mutant OCT1 using ANOVA followed by Tukey's honestly significant difference post hoc comparisons in SPSS Statistics version 25 (SPSS Inc., IBM, Chicago, IL).

\section{Results}

Characterization of the Model System. In this study, we used HEK293 cells stably transfected to overexpress human and mouse OCT1 by targeted chromosomal integration. As a first step, we characterized our model system with respect to the levels of OCT1 protein expression. We used targeted proteomics to quantify OCT1 expression in the stably transfected HEK293 cells and to compare it with OCT1 expression in human and mouse liver (Fig. 1). In HEK 293 cells, OCT1 expression was $36 \%$ higher in the cells stably overexpressing human compared with the cells stably overexpressing mouse OCT1 (Fig. 1A). The native OCT1 expression in human and mouse liver was similar but more than 10 -fold lower than in the model cell lines (16-fold for human OCT1, 11-fold for mouse OCT1, Fig. 1B). We also compared the OCT1 expression in three mouse strains: C57BL/6 substrains $\mathrm{J}$ and $\mathrm{N}$ and FVB. C57BL/6J mice showed more than $30 \%$ higher expression than the other two strains (Supplemental Fig. 1).

Differences in the Kinetics of Metformin Uptake between Human and Mouse OCT1. More importantly, we compared the uptake of metformin between human and mouse OCT1. To this end, we performed concentration-dependent uptake measurements in stably transfected HEK293 cells. The maximal transport rates $\left(\mathrm{v}_{\max }\right)$ were $45 \%$ lower in human than in mouse OCT1 ( $\mathrm{v}_{\max }$ of 939 and $1353 \mathrm{pmol} \times \min ^{-1} \times$ pmol OCT1 ${ }^{-1}$, respectively). The differences in the apparent affinity $\left(\mathrm{K}_{\mathrm{M}}\right)$ for metformin were much stronger (Fig. 2A). Mouse OCT1 showed a 4.9-fold higher affinity for metformin than human OCT1 $\left(\mathrm{K}_{\mathrm{M}}\right.$ of 491 and $2197 \mu \mathrm{M}$, respectively, $\left.P<0.0001\right)$. This resulted in a 6.5-fold higher intrinsic clearance of mouse compared with human OCT1 (2.86 and $0.50 \mu \mathrm{l} \times \min ^{-1} \times \operatorname{pmol~OCT}^{-1}$, respectively). The strong differences in metformin kinetics were confirmed when using a transient transfection model (Supplemental Fig. 2; Supplementary Table 4). In transiently transfected HEK293 cells, we observed an 8.1fold higher affinity of mouse OCT1 compared with human OCT1, resulting in a 12.5-fold difference in intrinsic clearance. Also, timedependent analyses showed substantially higher uptake by mouse than by human OCT1 (Fig. 2B). The difference was strongest in the beginning of the incubation period (5.7-fold at 1 minute) but remained above $60 \%$ even after 30 minutes of incubation. There were no indications for different modes of transport between human and mouse OCT1 (Fig. 2C). After incubating with clinically relevant concentrations of $10 \mu \mathrm{M}$ metformin (Shu et al., 2007), we observed 4.4-fold higher intracellular concentrations in HEK293 cells overexpressing mouse than in those overexpressing human OCT1 (99.6 and $22.7 \mu \mathrm{M}$, respectively, Fig. 2D).

We also determined the transport kinetics of rat OCT1 (Supplemental Fig. 3). With a $\mathrm{K}_{\mathrm{M}}$ of $422 \mu \mathrm{M}$ and intrinsic clearance of $30.5 \mu \mathrm{l} \times \min ^{-1} \times \mathrm{mg}_{\text {protein }}{ }^{-1}$, the rat ortholog did not differ significantly from mouse OCT1 $\left(\mathrm{K}_{\mathrm{M}}\right.$ of $491 \mu \mathrm{M}$ and intrinsic clearance of $37 \mu \mathrm{l} \times \mathrm{min}^{-1} \times \mathrm{mg}$ protein $\left.{ }^{-1}\right)$ but differed strongly compared with human OCT1 $\left(\mathrm{K}_{\mathrm{M}}\right.$ of $2197 \mu \mathrm{M}$ and intrinsic clearance of $7.85 \mu \mathrm{l} \times \min ^{-1} \times \mathrm{mg}$ protein $\left.{ }^{-1}\right)$.

Estimation of the Differences in Partition Coefficients of Metformin between Human and Mouse Livers Using IVIVE. Assuming that OCT1 is the major determinant of metformin levels in the liver (Wang et al., 2002; Shu et al., 2007), it could be expected that
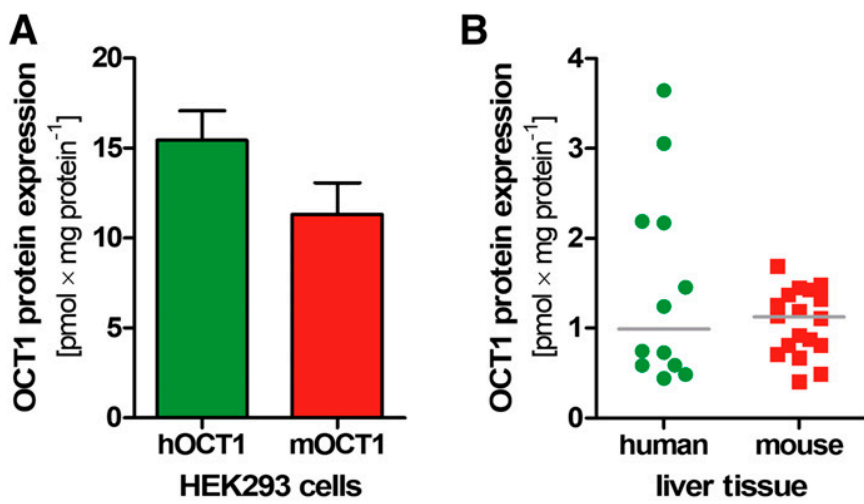

Fig. 1. OCT1 protein expression in (A) stably transfected HEK293 cells and (B) human and mouse liver. OCT1 expression in the membrane fraction of (A) HEK293 cells stably overexpressing human (green) or mouse (red) OCT1 or (B) human and mouse liver samples was measured by targeted LC-MS/MS. Please consider the difference in scaling of the $y$-axis. Shown are (A) means and S.E.M. of nine samples each and (B) concentrations of single samples (12 human and 20 mouse livers) and respective medians. 
A

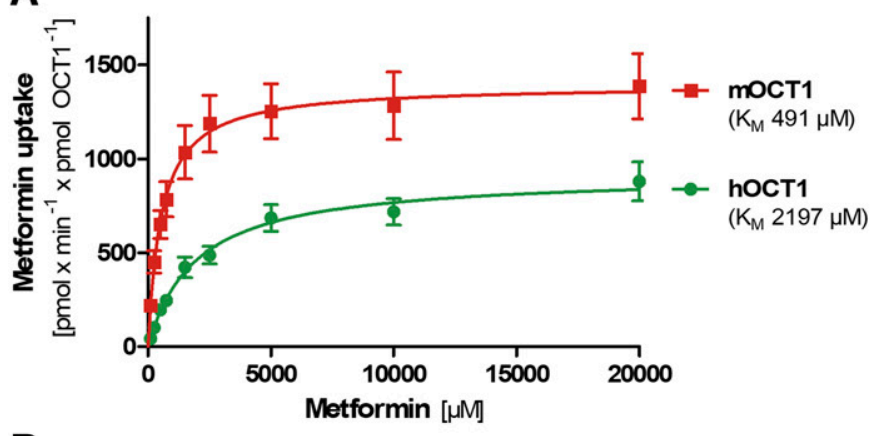

B

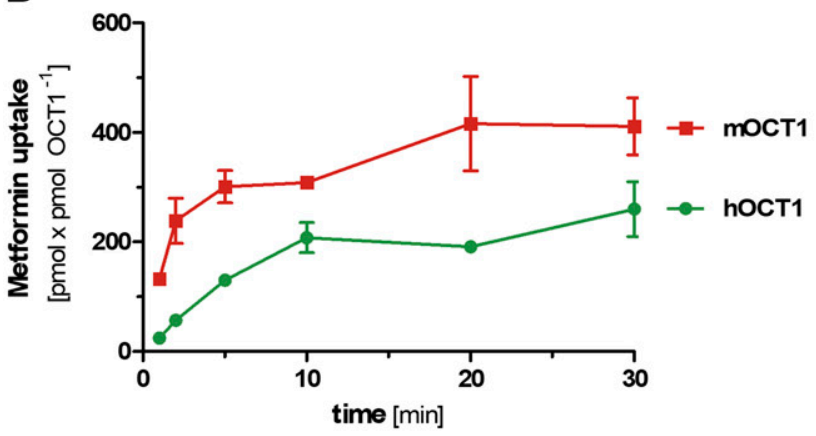

C

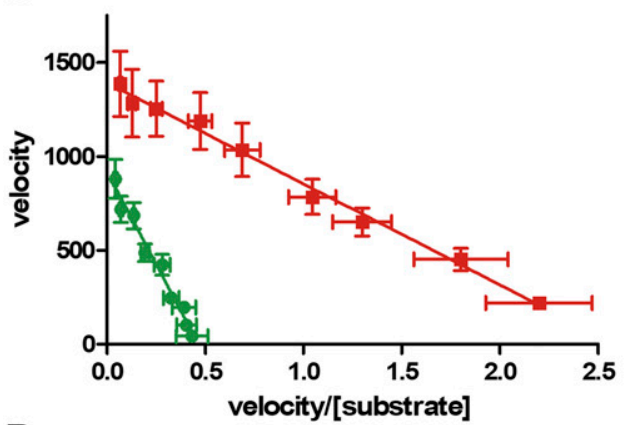

D

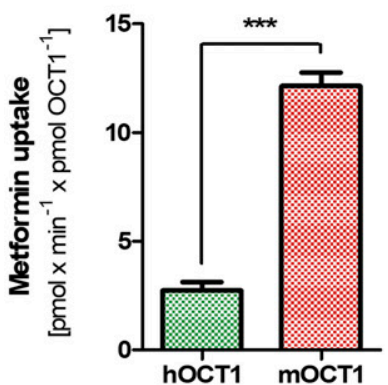

Fig. 2. Differences in metformin uptake between human and mouse OCT1. (A) Concentration-dependent uptake and (B) time-dependent uptake of metformin by human (green) and mouse (red) OCT1. OCT1-overexpressing HEK293 cells were incubated with (A) increasing concentrations of metformin for 2 minutes or (B) with $100 \mu \mathrm{M}$ metformin for up to 30 minutes. The uptake values were normalized to the amount of OCT1 protein in the respective HEK 293 cells, as determined by targeted proteomics (see Fig. 1A). (C) Eadie-Hofstee transformation of the data in (A). (D) Intracellular metformin concentrations in HEK293 cells stably transfected with human or mouse OCT1 after incubation with $10 \mu \mathrm{M}$ metformin for 2 minutes. The intracellular concentrations were calculated assuming an intracellular volume of 1.2 $\mu \mathrm{l}$ for $1 \times 10^{6}$ HEK293 cells, following the estimations of Chien et al. (2016). All subfigures represent OCT1-mediated uptake that was calculated by subtracting the uptake of control cells (pcDNA5) from the uptake of cells overexpressing OCT1. Shown are means and S.E.M. of at least three independent experiments. $* * * P<0.001$ in a one-way ANOVA. substantial differences in OCT1 clearance between human and mouse will result in substantial differences in the exposure to metformin in human and mouse liver. We used an IVIVE approach to estimate the $\mathrm{K}_{\mathrm{p}}$ both in human and in mouse. Taking into account the differences in OCT1 expression (Fig. 1), we estimated the liver-to-blood $\mathrm{K}_{\mathrm{p}}$ in human to be 3.34 compared with 14.4 in mouse (Table 2). Considering the estimated portal vein concentration of metformin in humans (Shu et al., 2007; Gormsen et al., 2016) and in mice (Wilcock and Bailey, 1994), we could expect 11-fold higher maximal intrahepatic concentrations in mice than in humans $(66.9 \mu \mathrm{M}$ in human and $746 \mu \mathrm{M}$ in mouse, Table 2).

We compared the predicted values for mouse with the experimentally measured values (Wilcock and Bailey, 1994). The predicted $K_{p}$ for mouse liver was almost 2-fold higher than the experimentally measured one (Table 2). In line with this, the predicted hepatic concentration in mouse was $98 \%$ higher. This suggests that our model overestimates the $\mathrm{K}_{\mathrm{p}}$ in mice and that factors other than OCT1-mediated uptake may play a role.

To the best of our knowledge, there is no experimental data on hepatic concentrations of metformin in humans. However, using ${ }^{11} \mathrm{C}$-labeled metformin in PET analyses, Gormsen et al. (2016) estimated a hepatic $\mathrm{K}_{\mathrm{p}}$ of 2.5 in humans, which is $34 \%$ lower than our estimation (Table 2) and supports the differences in hepatic metformin concentrations in vivo between human and mouse that were suggested by the IVIVE model.

Identification of the Structural Causes for the Differences in Metformin Kinetics. Next, we looked for structural differences between human and mouse OCT1 that confer the differences in their affinity for metformin. To this end, we generated chimeric constructs of human and mouse OCT1 (hmhOCT1 and mmhOCT1) and characterized their metformin uptake. We separated the protein into three parts: from $\mathrm{N}$ terminus to the large intracellular loop, from TMH7 to TMH9, and from TMH10 to $\mathrm{C}$ terminus (Fig. 3A). Concentrationdependent measurements pointed to the first six TMHs of OCT1 to confer the differences in affinity for metformin between human and mouse OCT1 (Fig. 3, B and C). Immunofluorescence staining demonstrated the correct membrane localization of the wild types and chimeras and suggested a reduced total expression of the mmhOCT1 chimera (Fig. 3D) that correlates with its reduced $\mathrm{v}_{\max }$ (Fig. 3B).

To further narrow down the region within the first six TMHs conferring these differences, we generated chimeric constructs with single TMH substitutions between human and mouse OCT1. Uptake experiments at single concentrations pointed to $\mathrm{TMH} 2$ and $\mathrm{TMH} 3$ as being primarily involved. Substituting TMH2 or TMH3 in human OCT1 with TMH 2 or TMH3 of mouse OCT1 resulted in the only significant increase of metformin uptake (Fig. 4A). In line with this, substituting TMH2 or TMH3 in mouse OCT1 with TMH2 or TMH3 of human OCT1 resulted in the strongest decrease of metformin uptake (by $77 \%$ and $55 \%$, respectively; Fig. 4B). A single concentration of $100 \mu \mathrm{M}$ was chosen to optimally reflect the difference in the $\mathrm{K}_{\mathrm{M}}$ based on the data from the wild-type constructs (Fig. 2). However, the effects (especially reduction of the uptake) may also be caused by a general reduction of activity (as observed for the mmhOCT1 chimera, Fig. 3B). To exclude this, we performed concentration-dependent measurements for TMH2- and TMH3-containing chimeras. We observed a strong increase in metformin affinity upon introduction of either mouse TMH2 or mouse TMH3 into human OCT1 (3-fold lower $\mathrm{K}_{\mathrm{M}}$ compared with human OCT1, Fig. 4C). Vice versa, introduction of either human TMH2 or human TMH3 into mouse OCT1 did not significantly change affinity. However, simultaneous introduction of human TMH2 and TMH3 into mouse OCT1 resulted in a significantly decreased affinity (14fold higher $\mathrm{K}_{\mathrm{M}}$ compared with mouse OCT1, Fig. 4C), and the concentration-dependent uptake almost completely mimicked the uptake of human OCT1 (Fig. 4D). These experiments clearly 
TABLE 2

Parameter of OCT1-mediated metformin pharmacokinetics in humans and mice measured experimentally or extrapolated using IVIVE

\begin{tabular}{|c|c|c|c|c|c|c|c|c|c|c|}
\hline \multirow{3}{*}{$\begin{array}{l}\text { Parameter } \\
\text { Maximal velocity, } \mathrm{v}_{\max }\left(\mathrm{pmol} \times \min ^{-1} \times \mathrm{mg}_{\text {protein }}{ }^{-1}\right)\end{array}$} & \multicolumn{5}{|c|}{ Mouse } & \multicolumn{5}{|c|}{ Human } \\
\hline & \multirow{2}{*}{$\begin{array}{c}\text { Mean } \\
17,496\end{array}$} & \multirow{2}{*}{$\frac{n}{11}$} & \multirow{2}{*}{$\begin{array}{c}\text { S.D. } \\
7097\end{array}$} & \multicolumn{2}{|c|}{$95 \% \mathrm{CI}$} & \multirow{2}{*}{$\begin{array}{c}\text { Mean } \\
14,703\end{array}$} & \multirow{2}{*}{$\frac{n}{11}$} & \multirow{2}{*}{$\frac{\text { S.D. }}{4346}$} & \multicolumn{2}{|c|}{$95 \% \mathrm{CI}$} \\
\hline & & & & 12,727 & 22,265 & & & & 11,783 & 17,623 \\
\hline Affinity for metformin uptake, $K_{M}(\mu \mathrm{M})$ & 491 & 11 & 155 & 387 & 595 & 2198 & 11 & 1154 & 1422 & 2973 \\
\hline Metformin in vitro clearance, $\mathrm{CL}_{\mathrm{in} \text { vitro }}\left(\mu \mathrm{l} \times \min ^{-1} \times \mathrm{mg}\right.$ protein $\left.{ }^{-1}\right)$ & 37 & 11 & 16.1 & 26.2 & 47.9 & 7.85 & 11 & 3.9 & 5.23 & 10.5 \\
\hline Metformin passive diffusion, $\mathrm{CL}_{\mathrm{diff}}\left(\mu \mathrm{l} \times \min ^{-1} \times \mathrm{mg}_{\text {protein }}{ }^{-1}\right)^{a}$ & 0.34 & 11 & 0.20 & 0.21 & 0.48 & 0.34 & 11 & 0.20 & 0.21 & 0.48 \\
\hline OCT1 expression in liver, $\mathrm{E}_{\text {in vivo }}\left(\mathrm{pmol} \times \mathrm{mg}\right.$ protein $\left.^{-1}\right)$ & 1.27 & 20 & 0.72 & 0.93 & 1.61 & 1.44 & 12 & 1.09 & 0.75 & 2.13 \\
\hline OCT1 expression in vitro, $\mathrm{E}_{\text {in vitro }}\left(\mathrm{pmol} \times \mathrm{mg}\right.$ protein $\left.^{-1}\right)$ & 11.3 & 9 & 5.23 & 7.3 & 15.3 & 15.4 & 9 & 4.92 & 11.7 & 19.2 \\
\hline Predicted metformin liver-to-blood partition coefficient, $\mathrm{K}_{\mathrm{p}, \mathrm{u}}{ }^{b}$ & 14.4 & 11 & 4.93 & 11.1 & 17.7 & 3.34 & 11 & 0.98 & 2.68 & 4.0 \\
\hline Predicted maximal hepatic metformin concentrations $(\mu \mathrm{M})^{c}$ & 746 & 11 & 254 & 575 & 659 & 66.9 & 11 & 19.6 & 53.7 & 80.0 \\
\hline Observed metformin $\mathrm{K}_{\mathrm{p}, \mathrm{u}}{ }^{d}$ & 6.8 & & & & & 2.5 & & & & \\
\hline Observed maximal hepatic metformin concentrations $(\mu \mathrm{M})^{d}$ & 350 & & & & & & & & & \\
\hline
\end{tabular}

S.D., standard deviation; $n$, number of independent measurments; CI, confidence interval.

${ }^{a}$ Passive diffusion was estimated based on the uptake in control HEK293 cells transfected with the empty vector pcDNA5 (Supplemental Fig. 2A). Therefore, the values do not differ between mouse and human.

${ }^{b} \mathrm{k}_{\mathrm{p}, \mathrm{u}}$ was calculated as described in Materials and Methods. Liver weights used for the calculations were $1500 \mathrm{~g}$ for human and $2.5 \mathrm{~g}$ for mouse (Rogers and Dintzis, 2018). The total amount of protein was 90 and $115 \mathrm{mg} \times \mathrm{g} \mathrm{liver}^{-1}$ for human and mouse liver, respectively (Sohlenius-Sternbeck, 2006).

${ }^{c}$ Hepatic concentrations were calculated assuming portal vein concentrations of $51.7 \mu \mathrm{M}$ for mouse (Wilcock and Bailey, 1994) and 20 $\mu \mathrm{M}$ for human [double the $C_{\mathrm{max}}$ observed in humans after $1 \mathrm{~g}$ of metformin (Shu et al., 2007; Gormsen et al., 2016)].

${ }^{d}$ The experimental data of mouse $\mathrm{k}_{\mathrm{p}}$ and hepatic concentrations were obtained from Wilcock and Bailey (1994) $30 \mathrm{~min}$ after an oral dose of $50 \mathrm{mg} / \mathrm{kg}$ metformin. The concentrations were calculated

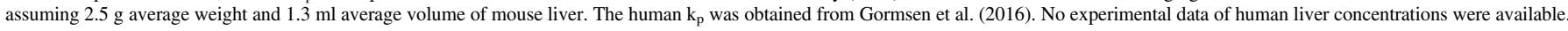

identify TMH2 and TMH3 to confer the differences between human and mouse OCT1 in their affinity for metformin.

Next, we generated homology models of human and of mouse OCT1 to identify single amino acids within TMH2 and TMH3 that may confer the differences in metformin uptake. There were no major differences in the tertiary structure between human and mouse OCT1 as visible by superposition of the two models (Fig. 5A). Two major binding cavities with the involvement of TMH 2 or TMH3 were identified using two distinct algorithms. One of the proposed binding cavities is located in the middle of the translocation pore and is a highly populated site with probe molecules (143 alpha spheres for human OCT1 and 120 alpha spheres for mouse OCT1). This "classical" binding site has been reported in several previous studies (Chen et al., 2017; Boxberger et al., 2018; Gorboulev et al., 2018). The binding site is enframed by TMH1, TMH4, TMH5, TMH7, TMH8, TMH9, TMH11, and (more importantly) TMH2. None of the five nonconserved amino acids in TMH2 (Fig. 5B; Supplemental Fig. 4) could be suggested to be directly involved in substrate binding. However, our structural models show that Leu155 in human (hLeu155) that corresponds to Val156 in mouse OCT1 (mVal156) in TMH2 can form a hydrophobic core packing with Ile35 located in TMH1 (Fig. 6, A and B) that may have an impact on tertiary structure stability (the mouse OCT1 protein is longer than the human one, resulting in a one-count shift in amino acid position after number 84). The stronger hydrophobic interaction between Leu155 and Ile35 in human OCT1 (compared with Val156 and Ile35 in mouse OCT1) might aggravate the entrance of substrates and conformational changes in this region of the transporter. These observations might explain a generally lower affinity for metformin uptake in human OCT1 compared with mouse OCT1.

Indeed, simultaneous introduction of mouse TMH3 and mutation of Leu $155 \mathrm{Val}$ increased the affinity of human OCT1 by more than $70 \%$ (of the difference between human and mouse OCT1, Fig. 6C), and vice versa, simultaneous introduction of human TMH3 and mutation of Val156Leu decreased metformin affinity in mouse OCT1 by $55 \%$ (Fig. 6, C and D). Without the simultaneous introduction of TMH3, the mutation of Leu155Val in human showed only limited effects, and Val156Leu in mouse OCT1 showed no significant effects. This points to the importance of the interaction with TMH3 for the effects of hLeu155/mVal156.

An alternative explanation involving both TMH2 and TMH3 may be provided by the second predicted binding site. This binding cavity (although with a lower score, as indicated by 51 alpha spheres for human OCT1 and 25 alpha spheres for mouse OCT1; Fig. 7, A and B, left panel) is a membrane-exposed pocket with involvement of residues from both TMH2 and TMH3. Interestingly, this "outer" cavity is framed by two nonconserved residues_one from each $\mathrm{TMH}$ - lying just opposite of each other. Whereas in mouse OCT1, these residues are valines (Val166 and Val182), in human OCT1, these residues are glycines (Gly165 and Gly181; Fig. 7, A and B). The glycines in human OCT1 could lead to a higher conformational flexibility of the helices in that region, whereas the valines in mouse OCT1 introduce hydrophobicity and potentially stronger interactions with ligands at this position. Indeed, mutation of valines 166 and 182 in mouse OCT1 to glycines (Val166Gly, Val182Gly), either alone or in combination, significantly decreased metformin uptake (Fig. 7C). However, the decrease was maximally 26\%, and the reverse mutation of glycines in human OCT1 to valines (Gly165Val, Gly181Val) neither alone nor in combination affected metformin uptake. This suggests that the glycine-to-valine differences at positions 165/166 and 181/182 alone cannot explain the differences in metformin uptake between human and mouse OCT1.

As an alternative approach, we took advantage of the observation that the affinity of human OCT2 for metformin is rather similar to the affinity of mouse OCT1 than to the affinity of human OCT1 (Supplemental Fig. 5A). We mutated amino acids that are identical in mouse OCT1 and human OCT2 but are different in human OCT1 (Supplemental Fig. 5B) and analyzed the effects on metformin uptake. In addition to hLeu155/ mVal156, which we already analyzed (Fig. 6C), this affected hPhe169/ mIle170 in TMH2. Interestingly, this variation is the only difference between human and mouse OCT1 within the conserved A-motif (Fig. 5B), which is suggested to be important for both structure and function of MFS transporters by interacting with residues from surrounding TMHs and supporting conformational changes during the transport cycle (Henderson and Maiden, 1990; Pao et al., 1998; Quistgaard et al., 2016). Mutation of Ile170 in mouse OCT1 to the corresponding amino acid in human OCT1 (Ile170Phe) decreased metformin uptake by $28 \%\left(P=4 \times 10^{-4}\right.$, Supplemental Fig. 5C). However, mutation of both Val156Leu and Ile170Phe in mouse OCT1 did not lead to a stronger decrease in metformin uptake than mutation of Val156Leu alone (43\%, Supplemental Fig. 5C). Mutation of these amino acids in human OCT1 (Leu155Val and Phe169Ile) neither alone nor in combination had an effect on metformin uptake (Supplemental Fig. 5C). Thus, hPhe169/mIle170 neither alone nor 
A

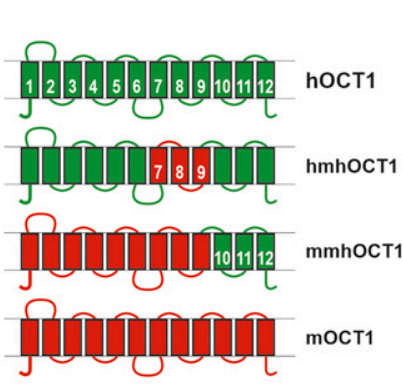

B

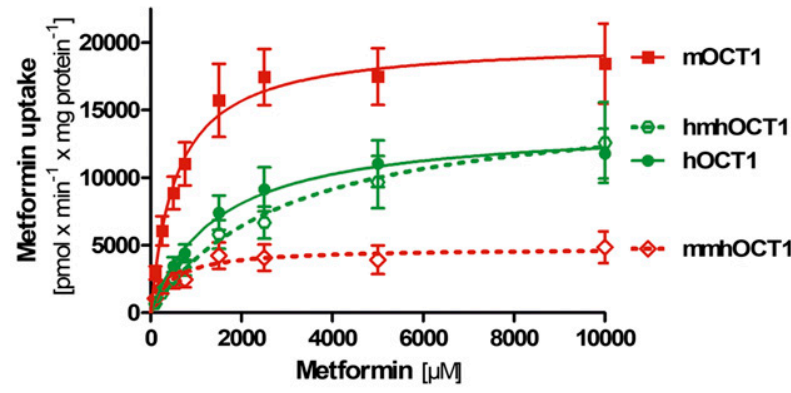

C

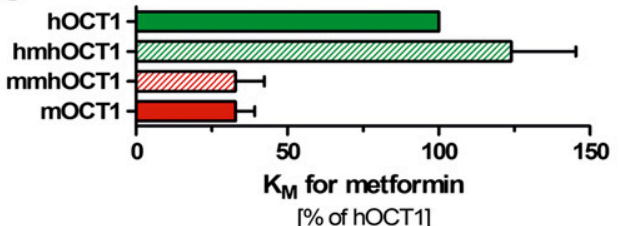

D antibody

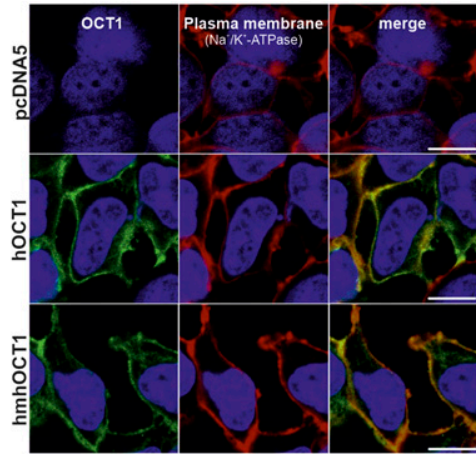

antibody

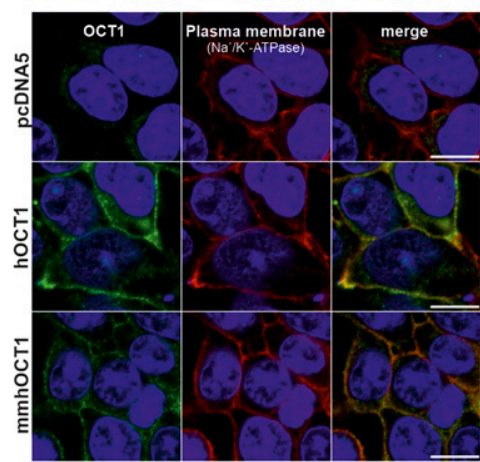

antibody
mOCT1

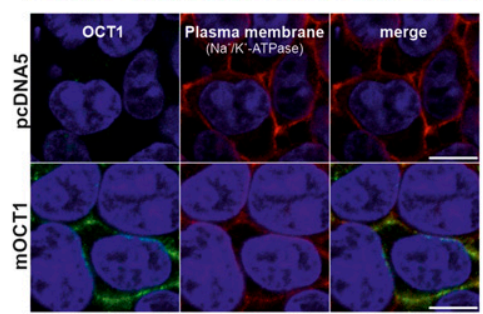

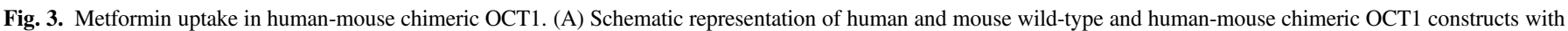

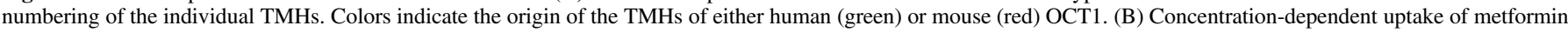

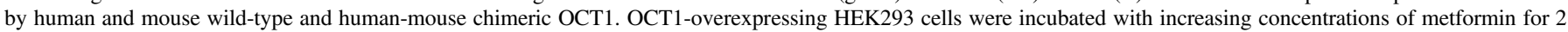

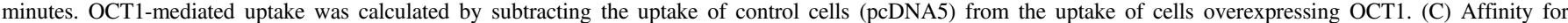

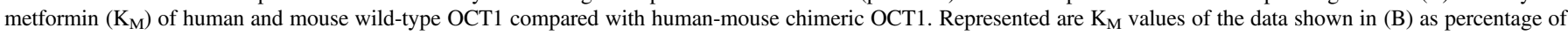

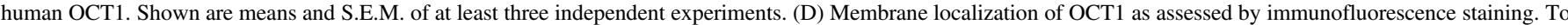

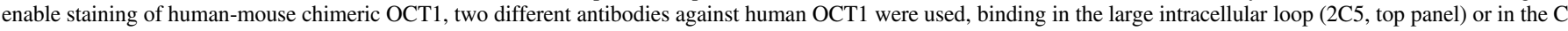

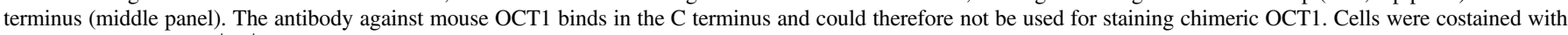
an antibody against $\mathrm{Na}^{+} / \mathrm{K}^{+}$-ATPase as a marker for the plasma membrane. Cell nuclei were stained with DAPI. Scale bar, $10 \mu \mathrm{m}$.

in combination with hLeu155/mVal156 can explain more than $30 \%$ of the observed differences in metformin affinity between the species. In addition, this suggests that independent mechanisms confer the higher affinity for metformin in mouse OCT1 and in human OCT2.

Differences in the Kinetics of Thiamine Uptake between Human and Mouse OCT1. Similar to metformin, we observed strong differences in the affinity for thiamine between human and mouse OCT1 (Fig. 8). Mouse OCT1 had a 9.5-fold higher apparent affinity for thiamine than human OCT1 ( $\mathrm{K}_{\mathrm{M}}$ of 143 and $1057 \mu \mathrm{M}$, respectively; Fig. 8A). In contrast to metformin, the lower affinity for thiamine of human OCT1 resulted in $80 \%$ higher maximal transport rates $\left(\mathrm{v}_{\max }\right.$ of 528 and $287 \mathrm{pmol}$ $\times \min ^{-1} \times$ pmol OCT1 ${ }^{-1}$ for human and mouse OCT1, respectively). Nevertheless, this resulted in a 5.1-fold higher intrinsic clearance of thiamine by mouse OCT1 compared with human OCT1 (Fig. 8C).

Concentration-dependent measurements using human-mouse chimeric OCT1 also pointed to the first six TMHs of OCT1 to confer the differences in affinity for thiamine between human and mouse OCT1 (Fig. 9, A and B). Considering the observed key role of TMH2 and TMH3 in the transport of metformin, we analyzed thiamine uptake by human-mouse chimeric OCT1 carrying the simultaneous substitution of both helices. Similar to metformin, simultaneous introduction of mouse TMH2 and TMH3 into human OCT1 resulted in a significant increase of affinity for thiamine ( $\mathrm{K}_{\mathrm{M}}$ of $456 \mu \mathrm{M}$ compared with $1517 \mu \mathrm{M}$ of human OCT1, Fig. 9, C and D). However, in contrast to metformin, introduction of human TMH2 and TMH3 into mouse OCT1 did not result in a significant decrease of affinity (Fig. 9C). Therefore, it could be concluded that TMH2 and TMH3 are also involved in the mechanisms conferring differences in thiamine uptake between human and mouse OCT1, but the mechanisms are not identical to the ones for metformin.

\section{Discussion}

In this study, we report strong differences between human and mouse OCT1 in the transport of metformin and thiamine. The most pronounced difference was the substantially higher apparent affinity of mouse compared with human OCT1, which results in a much higher intrinsic uptake clearance. This was observed for both metformin and thiamine. As a consequence, higher concentrations of metformin may be reached in mouse than in human liver. Furthermore, differences in the affinity for metformin between human and mouse OCT1 could be attributed to 


\section{Uptake at single concentration}

A

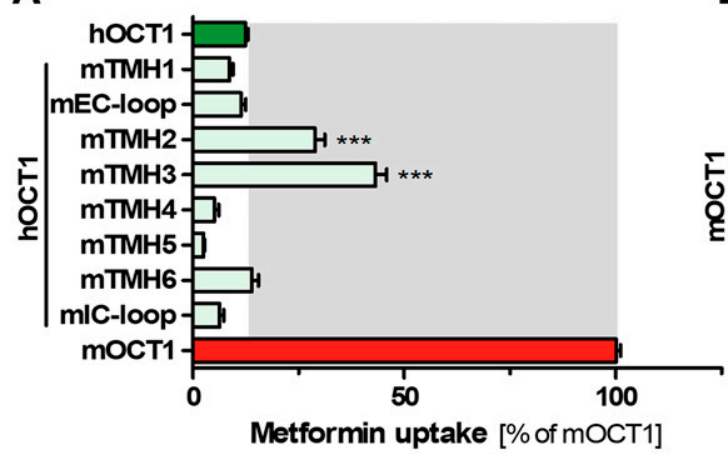

B

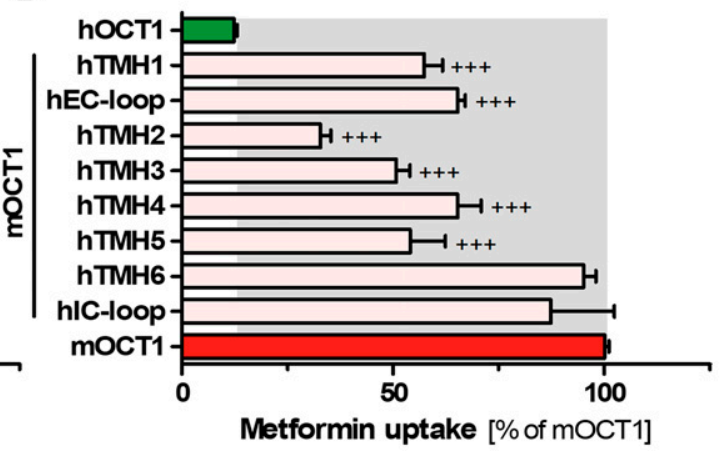

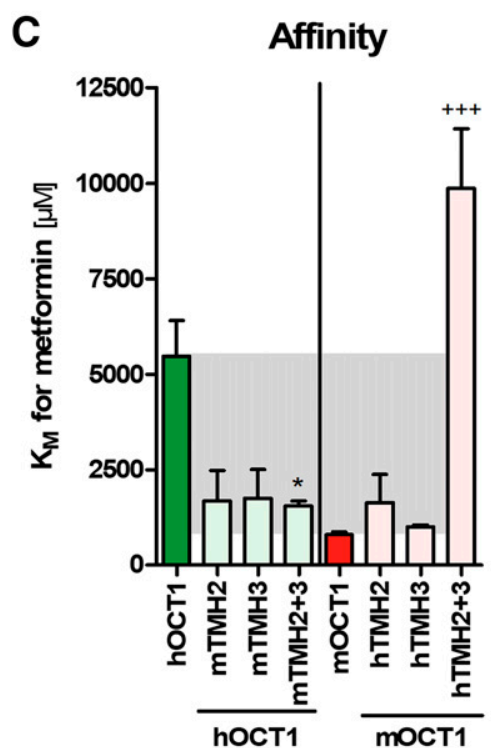

D

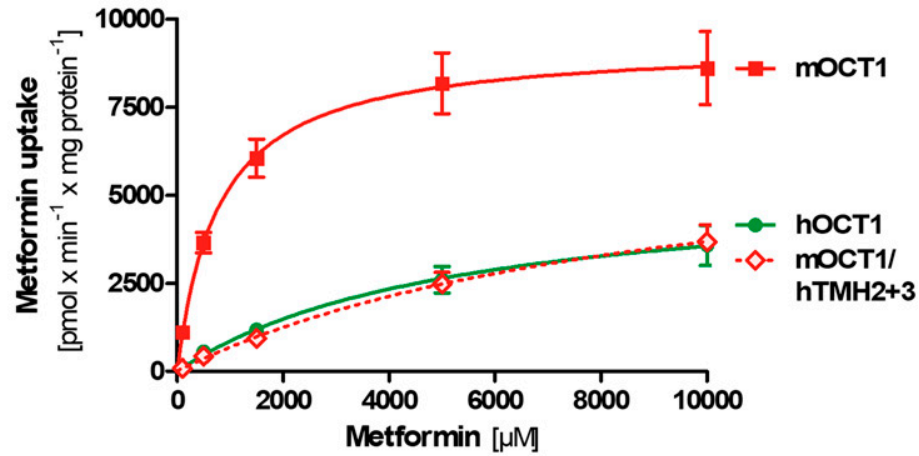

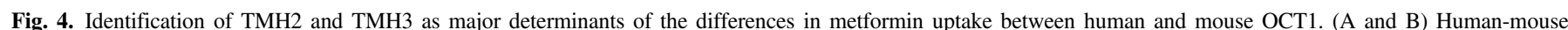

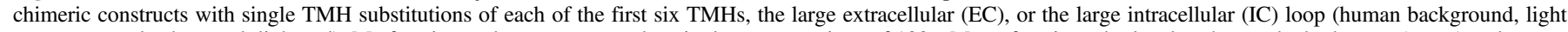

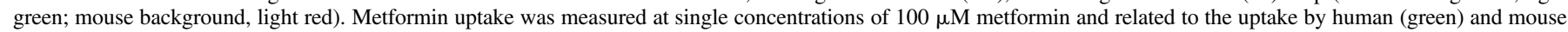

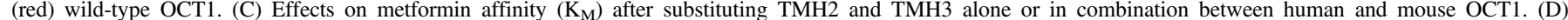

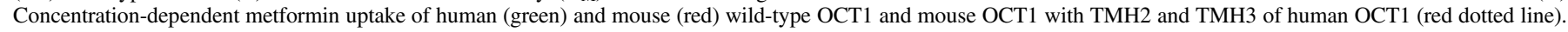

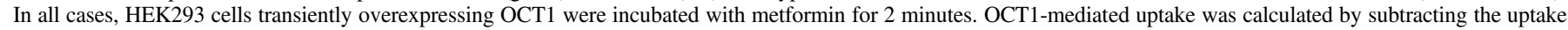

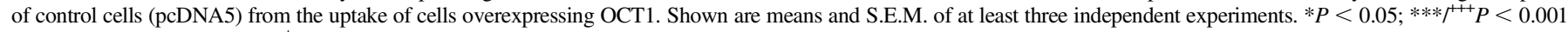
compared with $\left(^{*}\right)$ human or $\left(^{+}\right)$mouse OCT1 in a Tukey's post hoc analysis following one-way ANOVA.

differences in TMH 2 and 3 of the transporter, revealing new insights into the transport mechanism of metformin by OCT1.

We observed a 4.9-fold higher affinity for metformin by mouse than by human OCT1 and a $45 \%$ higher transport capacity (Fig. 2). The affinity of human OCT1 for metformin observed here $\left(\mathrm{K}_{\mathrm{M}}\right.$ of $2197 \mu \mathrm{M}$, Fig. 2) is similar to previous reports (Shu et al., 2007; Umehara et al., 2007; Nies et al., 2009). Despite mouse being a commonly used model organism to study metformin pharmacokinetics and effects, to the best of our knowledge, this is the first study reporting metformin uptake kinetics via mouse OCT1. Moreover, we characterized metformin uptake by human and mouse OCT1 in parallel. Therefore, the obtained data are highly comparable, especially since we used a model system that is well characterized in terms of protein expression, enabling us to normalize uptake data to the amount of OCT1 protein. There are no previous reports on metformin uptake kinetics in mouse hepatocytes. However, comparison of human and rat hepatocytes showed a 27-fold higher metformin clearance in rats than in humans (Umehara et al., 2007), and we observed highly similar uptake kinetics between rat and mouse OCT1 (Supplemental Fig. 3).

Based on our in vitro data on OCT1 affinity and on the differences in the portal vein concentrations, metformin concentrations can be expected to be about 11-fold higher in mouse than in human liver (Table 2). This could result in differences in the hepatic actions of metformin between human and mouse. Low metformin concentrations were suggested to activate AMPK (Zhou et al., 2001) and to suppress gluconeogenic gene expression and glucose production (Cao et al., 2014), whereas high metformin concentrations inhibit mitochondrial complex I (El-Mir et al., 2000) and lead to an AMPK-independent suppression of gluconeogenesis (Foretz et al., 2010). Especially as OCT1-overexpression has recently been shown to substantially increase the mitochondrial accumulation of the drug (Chien et al., 2016), mitochondrial effects of metformin may be more likely in mouse than in human liver. In general, our data warrants attention when using mouse data to extrapolate the hepatic effects of metformin in humans. 


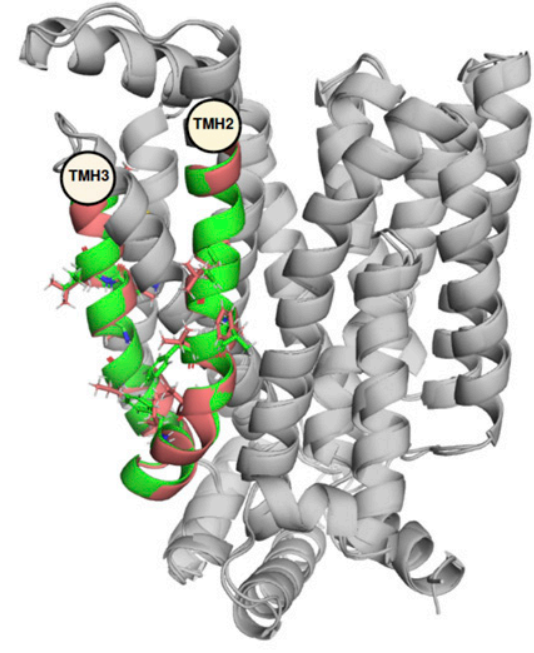

B

TMH2

TMH3

hOCT1 150 LF SCLNAGFFFGSLGVGYFADRFGRKLCLLGTVLVNAVSGVLMAFSP MOCT1 151 LF QSCVNLGFFLGSLVVGYIADRFGRKLCLLVTTLVTSLSGVLTAVAP $\uparrow$ $\Delta$ $\Delta$
Fig. 5. Structural differences between human and mouse OCT1 with a focus on TMH2 and TMH3. (A) Superposition of human and mouse OCT1 structural models with TMH2 and TMH3 highlighted in green (human OCT1) and red (mouse OCT1). (B) Protein sequence alignment of human and mouse OCT1 using EMBOSS Needle (Madeira et al., 2019) with TMH2 and TMH3 highlighted and the conserved A-motif of MFS transporters underlined in violet. Coloring is based on amino acid identity. Arrows indicate the positions of amino acids hLeu155/mVal156 (closed arrow) and hGly $165 / \mathrm{mVal166}$ and hGly181/mGly182 (open arrows), which were of particular interest.
Because of the better uptake by mouse OCT1, the liver-mediated effects may be more pronounced in mice than in humans.

The effects of OCT1 deficiency are more pronounced in mice than in humans (Wang et al., 2002, 2003; Shu et al., 2007; Zhou et al., 2009; Dujic et al., 2017; Sundelin et al., 2017). A 30-fold decrease in hepatic metformin concentrations was shown in OCT1 knockout mice (Wang et al., 2002). Precise measurements in humans are difficult, but a study using PET imaging showed about 2-fold lower hepatic metformin concentrations in carriers of loss-of-function OCT1 variants (Sundelin et al., 2017). These numbers generally fit to the tendency observed here that OCT1-mediated uptake is about 14-fold higher compared with diffusion in mice and only 3-fold higher compared with diffusion in humans (Table 2). One explanation, supported by our data, is that because of the different efficacy of human and mouse OCT1 in transporting metformin, knockout in mice and loss-of-function genetic variants in humans do not have comparable effects on hepatic metformin concentrations. However, the fact that some of the human OCT1 genetic variants do not lead to a complete loss of metformin uptake should also be considered (Kerb et al., 2002; Shu et al., 2003; Seitz et al., 2015).

Our IVIVE calculations based on the uptake kinetics of mouse OCT1 overestimated the hepatic $\mathrm{K}_{\mathrm{p}}$ and hepatic concentrations of metformin experimentally measured in mice (Table 2). The most probable reason for this is that the major reflection of the higher uptake clearance via mouse OCT1 is observed in the first minutes of the uptake (Fig. 2B), and the available experimental data are obtained after 30 minutes or more (Wilcock and Bailey, 1994). In the longer incubation, other factors, like reaching steady state of intracellular versus extracellular metformin concentrations, may play a role. Therefore, short-term differences in the concentrations of metformin between human and mouse liver may be more pronounced than the long-term differences. Nevertheless, accounting for the PET-based estimation of $\mathrm{K}_{\mathrm{p}}$ of 2.5 in humans and a maximal portal vein concentration of $20 \mu \mathrm{M}$ (Shu et al., 2007; Gormsen et al., 2016), an intrahepatic concentration of about $50 \mu \mathrm{M}$ could be estimated for the human liver. This is 7-fold lower than the intrahepatic concentrations of metformin measured in mice (Wilcock and Bailey, 1994).

The involvement of alternative transporters like OCT3 that are not reflected in our IVIVE calculations is less probable. In the mouse liver,
OCT1 has much higher expression levels than OCT3 [OCT1-to-OCT3 mRNA ratio of about 30 (Chen et al., 2015)]. Consistently, up to 30-fold lower hepatic metformin concentrations were measured in OCT1 knockout mice (Wang et al., 2002), but there were no significant changes in OCT3 knockout mice (Chen et al., 2015; Lee et al., 2018). Also, in the human liver, OCT1 is expressed much more strongly than OCT3. The ratio of OCT1 to OCT3 in human liver is 22 based on protein quantification (Drozdzik et al., 2019) and 32 based on mRNA quantification (Nies et al., 2009). The intrinsic clearance of metformin can thus be estimated to be at least 11-fold lower by OCT3 than by OCT1, indicating that OCT1 is the predominant uptake transporter of metformin both in the mouse and in the human liver.

Similar to metformin, the affinity for thiamine was much higher by mouse than by human OCT1 (9.5-fold lower $K_{M}$, Fig. 8), which is supported by a previous study (Chen et al., 2014). Also, clear effects of OCT1 deficiency on thiamine levels were reported in mice (Chen et al., 2014; Liang et al., 2018) but not in humans (Jensen et al., 2020). One explanation may be that in humans, at "physiological" low concentrations, thiamine is predominantly transported by thiamine transporters THTR-1 and THTR-2, which have a substantially higher affinity $\left(>1600\right.$-fold lower $\mathrm{K}_{\mathrm{M}}$ ) but also a substantially lower capacity $\left(>130\right.$-fold lower $\mathrm{v}_{\max }$ ) than OCT1 (Jensen et al., 2020). In contrast, in mice, the differences in affinity and capacity of thiamine uptake between OCT1 and THTR-1 are much smaller (2.9-fold lower $\mathrm{K}_{\mathrm{M}}$ and 7.6-fold lower $\mathrm{v}_{\max }$ ), and mouse OCT1 showed more than 5-fold higher thiamine uptake than mouse THTR-1 at low concentrations [100 nM; (Chen et al., 2014)]. This, together with the much higher affinity of mouse compared with human OCT1, suggests that OCT1 may play a more important role in thiamine uptake at low concentrations in mice than in humans and thereby may contribute to the different effects of OCT1 deficiency on thiamine plasma levels between these species. Alternatively, the strong renal OCT1 expression in mouse but not in human (Gorboulev et al., 1997; Zhang et al., 1997; Green et al., 1999; Schmitt et al., 2003) may contribute to the different effects of OCT1 deficiency on systemic thiamine concentrations in the two species. However, compensatory effects of OCT2, which is strongly expressed in the kidney, are probable (Chen et al., 2014). 

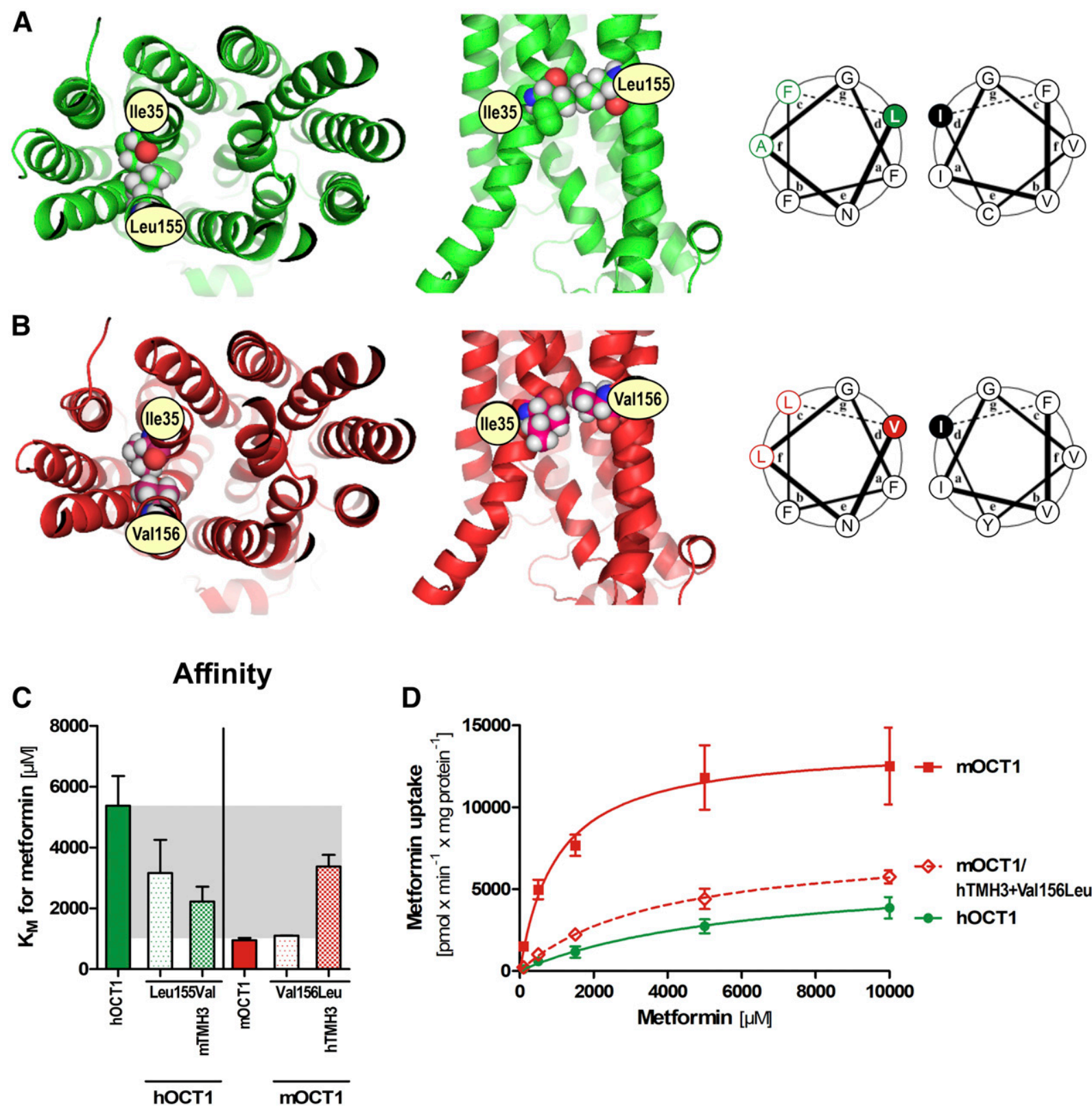

D

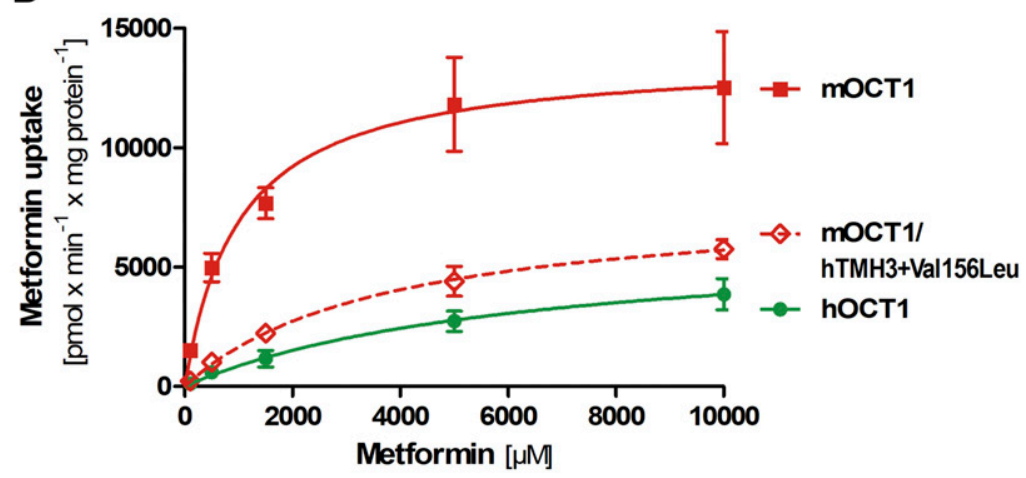

Fig. 6. Potential involvement of hLeu155/mVal156 in TMH2 of human/mouse OCT1 in conferring the differences in metformin affinity. Hydrophobic interactions between hLeu155/mVal156 and Ile35 in (A) human and (B) mouse OCT1 in (left panel) top view and (middle panel) side view. (A and B, right panel) Helical wheel projection of TMH2 and TMH1 showing the positioning of hLeu155/mVal156 (TMH2) and Ile35 (TMH1) in position "d" of the helical wheels, respectively. Nonconserved amino acids are highlighted in color, and Ile35 is highlighted in black. (C) Effects on metformin affinity $\left(\mathrm{K}_{\mathrm{M}}\right)$ after simultaneous substitution of Leu155Val and mouse TMH3 in human OCT1 and Val156Leu and human TMH3 in mouse OCT1. (D) Concentration-dependent metformin uptake of human (green) and mouse (red) wild-type OCT1 and mouse OCT1 with Val156Leu mutation and human TMH3 (red dotted line). In all cases, HEK293 cells transiently overexpressing OCT1 were incubated with metformin for 2 minutes. OCT1-mediated uptake was calculated by subtracting the uptake of control cells (pcDNA5) from the uptake of cells overexpressing OCT1. Shown are means and S.E.M. of four independent experiments. $* P<0.05, * * * f^{+++} P<0.001$ compared with $(*)$ human or $\left({ }^{+}\right)$mouse OCT1 in a Tukey's post hoc analysis following one-way ANOVA.

Transmembrane helices TMH2 and TMH3 were experimentally identified to confer the differences in metformin affinity between human and mouse OCT1. Three independent hypotheses were generated to identify the single amino acids responsible: 1) differences in the hydrophobic interaction of hLeu 155/mVal156 (TMH2) with Ile35 (TMH1), 2) higher flexibility by Gly 165 and Gly181 in human as opposed to higher hydrophobicity and ligand interaction by Val166 and Val182 in mouse OCT1, and 3) similar affinities of mouse OCT1 and human OCT2 caused by Val156 and/or Ile170 that differ in human OCT1 (Ile170 being located within the conserved
A-motif of the MFS transporters). Experimentally, the strongest effects were observed by mutating Val156 to Leu together with exchanging TMH3 (Fig. 6), supporting the first hypothesis the most. The mechanisms may be expected to be similar for rat OCT1, since the potentially involved amino acids are identical between rat and mouse OCT1 (Supplemental Fig. 3D).

Another interesting observation is that the substitution of a single TMH (either 2 or 3 ) is sufficient to increase the affinity of human OCT1, but both human TMHs are needed to decrease the affinity of mouse OCT1 (Fig. 4C). This may suggest that a decreased affinity requires an 
A

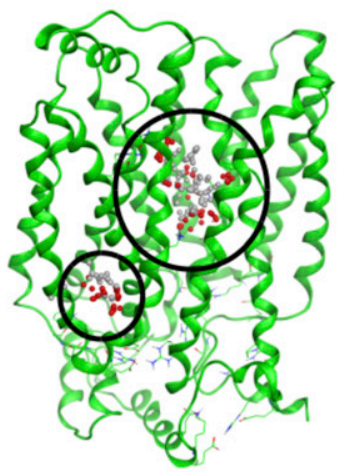

B

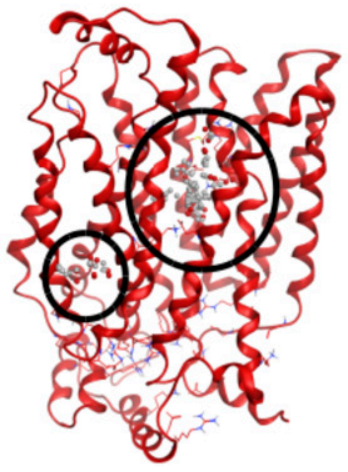

C

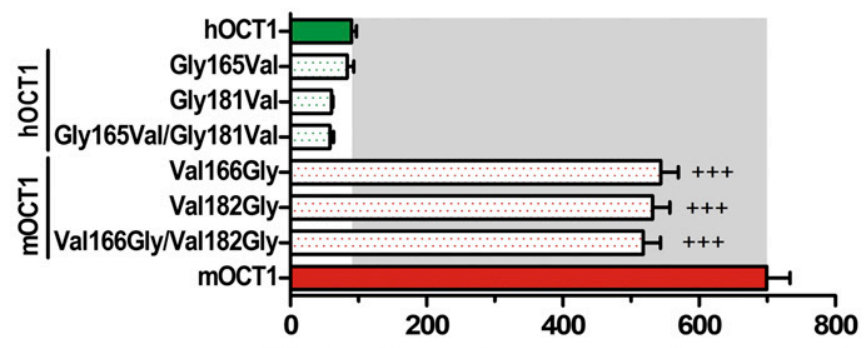

Metformin uptake $\left[\mathrm{pmol} \times \mathrm{min}^{-1} \times \mathrm{mg}_{\text {protein }}{ }^{-1}\right]$

Fig. 7. Potential involvement of TMH2 and TMH 3 in conferring the differences in metformin affinity between human and mouse OCT1. (A and B, left panel) Two predicted binding cavities within the TMH2-TMH3 region in (A) human and (B) mouse OCT1 structural models. (A and B, right panel) Top view of the "inner" binding cavity with protein surface colored according to lipophilicity (red, hydrophilic; yellow, hydrophobic; white, neutral surface) with (A) glycine residues 165 and 181 in human OCT1 and (B) valine residues 166 and 182 in mouse OCT1 highlighted. (C) Effect of mutations of Gly165Val and Gly181Val in human and Val166Gly and Val182Gly in mouse OCT1 on metformin uptake. HEK293 cells transiently overexpressing OCT1 were incubated with $100 \mu \mathrm{M}$ metformin for 2 minutes. OCT1-mediated uptake was calculated by subtracting the uptake of control cells (pcDNA5) from the uptake of cells overexpressing OCT1. Shown are means and S.E.M. of two to four independent experiments performed in duplicates. ${ }^{++} P<0.001$ compared with mouse OCT1 in a Tukey's post hoc analysis following one-way ANOVA.

interaction between the two TMHs and removing one of the TMHs is enough to destroy this interaction.

From the structural perspective, the "knob-into-hole" motif of the hydrophobic interaction between hLeu $155 / \mathrm{mVal} 156$ in TMH2 with Ile35 in TMH1 (Fig. 6) is somewhat analogous to coiled-coil structures (Liu et al., 2006). Interestingly, hLeu $155 / \mathrm{mVal} 156$ are located at position "d" when depicting the helix as a helical wheel projection (Fig. 6). Since this position is suggested to be more vulnerable to amino acid substitution (Zhu et al., 1993), hLeu155/mVal156 may have a huge impact on tertiary structure stability. The stronger hydrophobic interaction between Leu155 and Ile35 in human OCT1 (compared with Val156 and Ile35 in mouse OCT1) might obstruct substrate entry and conformational changes in this region, thereby potentially explaining the lower affinity for metformin.

Another aspect to be considered is the substrate-specific effects of OCT1. Recently, Morse et al. (2020) reported substantial differences in the uptake kinetics between human and mouse primary hepatocytes for ondansetron and tropisetron but not for sumatriptan and fenoterol. In our study, the differences in the affinity between human and mouse OCT1 were comparable between metformin and thiamine (Figs. 2 and 8). This is consistent with previous reports suggesting similar binding sites of metformin and thiamine in OCT1 (Chen et al., 2014). However, our data show that the affinities for these two compounds are conferred by similar, but not identical, structures in OCT1 (Figs. 4 and 9). This underlines the polyspecificity of OCT1 and points out that structure-tofunction relations of OCT1 need to be established separately for each substrate and, based on the present results, also for each species.

\section{A}

B
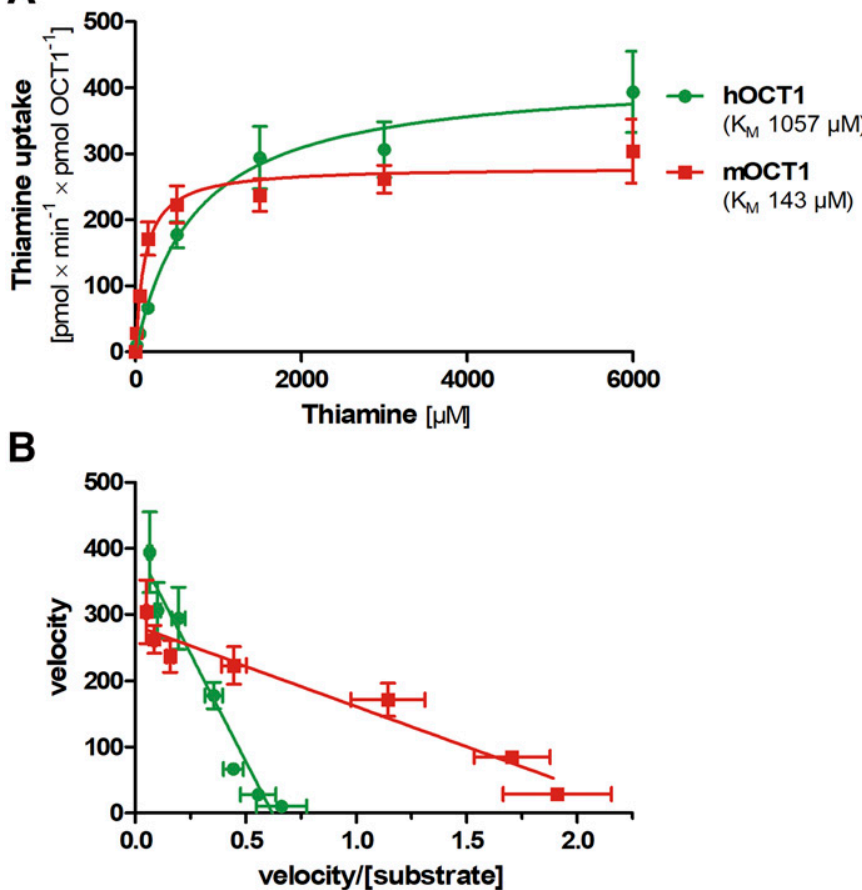

C

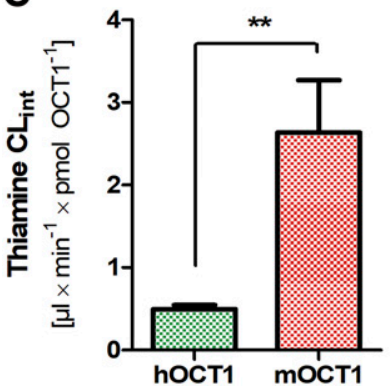

Fig. 8. Differences in thiamine uptake between human and mouse OCT1. (A) Concentration-dependent uptake of thiamine by human (green) and mouse (red) OCT1. OCT1-overexpressing HEK293 cells were incubated with increasing concentrations of thiamine for 2 minutes. OCT1-mediated uptake was calculated by subtracting the uptake of control cells (pcDNA5) from the uptake of cells overexpressing OCT1. Background thiamine levels were subtracted from all values to exclude influence of endogenous thiamine on the measurement. The uptake values were normalized to the amount of OCT1 protein in the respective HEK293 cells, as determined by targeted proteomics (see Fig. 1A). (B) EadieHofstee transformation of the data in (A). (C) Comparison of the intrinsic clearance $\left(\mathrm{CL}_{\text {int }}\right)$ between human and mouse OCT1 calculated using $\mathrm{v}_{\max }$ and $\mathrm{K}_{\mathrm{M}}$ of the data in (A). Shown are means and S.E.M. of at least three independent experiments. $* * P<0.01$ in a one-way ANOVA. 
A

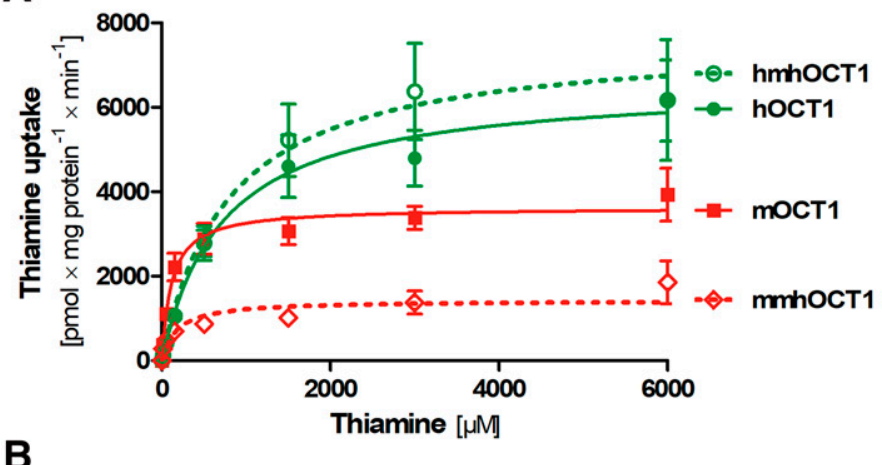

B

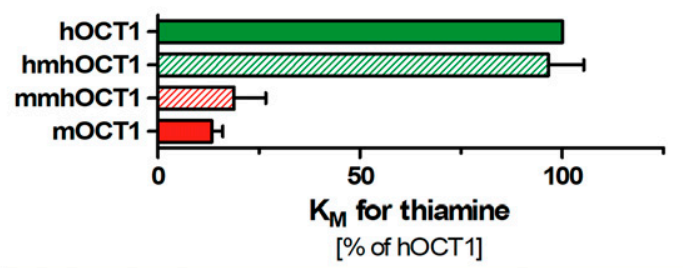

C

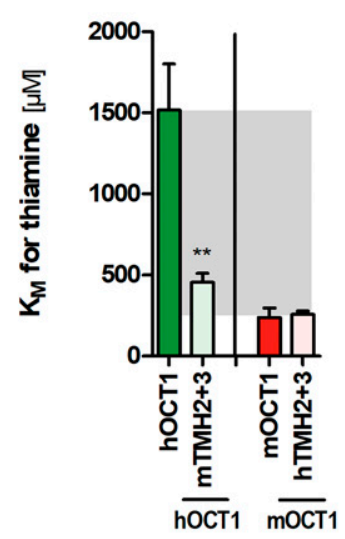

D

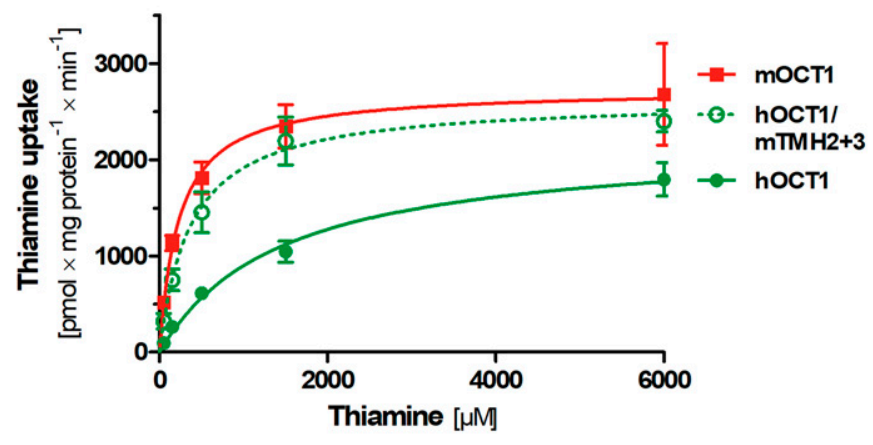

Fig. 9. Thiamine uptake in human-mouse chimeric OCT1 constructs. (A) Concentrationdependent uptake of thiamine by human and mouse wild-type and human-mouse chimeric OCT1. For detailed description of chimeric constructs see figure 3A. (B) Affinity for thiamine $\left(\mathrm{K}_{\mathrm{M}}\right)$ of human and mouse wild-type compared with human-mouse chimeric OCT1. Represented are $\mathrm{K}_{\mathrm{M}}$ values of the data shown in (A) as percentage of human OCT1. (C) Affinity for thiamine $\left(\mathrm{K}_{\mathrm{M}}\right)$ of human and mouse wild-type OCT1 compared with human-mouse chimeric OCT1 carrying the simultaneous substitution of TMH2 and TMH3. (D) Concentrationdependent thiamine uptake of human (green) and mouse (red) wild-type OCT1 and human OCT1 with TMH2 and TMH3 of mouse OCT1 (green dotted line). HEK293 cells (A and B) stably or (C and D) transiently overexpressing OCT1 were incubated with increasing concentrations of thiamine for 2 minutes. OCT1 mediated uptake was calculated by subtracting the uptake of control cells (pcDNA5) from the uptake of cells overexpressing OCT1. Background thiamine levels were subtracted from all values to exclude the influence of endogenous thiamine on the measurement. Shown are means and S.E.M. of at least three independent experiments. ${ }^{*} P P<0.01$ in a Tukey's post hoc analysis following one-way ANOVA.
In conclusion, mouse OCT1 has a much higher affinity for metformin and thiamine than human OCT1. This may be an important factor contributing to the substantially higher metformin concentrations measured in the mouse than in the human liver in vivo and should be considered when interpreting findings about the hepatic mechanism of action of metformin that are obtained in mouse models. The determinants of the differences in metformin affinity between human and mouse OCT1 are clearly located in TMH2 and TMH3 and comprise hLeu 155/mVal156 (TMH2) and amino acid(s) in TMH3. The underlying mechanism is probably complex, and the identification of the precise amino acids in TMH3 and additional protein structures in that region involved needs further investigation.

\section{Acknowledgments}

The authors would like to acknowledge Helen Massy for her contribution to the initial cloning of mouse and rat OCT1 orthologs and the generation of the first chimeric constructs. We also highly acknowledge the technical support of Kerstin Schmidt and Tina Sonnenberger (Greifswald) in the uptake measurements and cloning and Cornelia Willnow (Göttingen) for support in collecting the human liver samples.

\section{Authorship Contributions}

Participated in research design: Meyer, Brockmöller, Zdrazil, Tzvetkov.
Conducted experiments: Meyer, Tuerkova, Wenzel.

Contributed new reagents or analytic tools: Tuerkova, Römer, Seitz, Gaedcke, Zdrazil.

Performed data analysis: Meyer, Tuerkova, Römer, Wenzel, Oswald, Zdrazil, Tzvetkov.

Wrote or contributed to the writing of the manuscript: Meyer, Tuerkova, Römer, Oswald, Brockmöller, Zdrazil, Tzvetkov.

\section{References}

Abraham MJ, Murtola T, Schulz R, Páll S, Smith JC, Hess B, and Lindahl E (2015) GROMACS high performance molecular simulations through multi-level parallelism from laptops to supercomputers. SoftwareX 1-2:19-25 DOI: 10.1016/j.softx.2015.06.001.

Boxberger KH, Hagenbuch B, and Lampe JN (2018) Ligand-dependent modulation of hOCT1 transport reveals discrete ligand binding sites within the substrate translocation channel. Biochem Pharmacol 156:371-384 DOI: 10.1016/j.bcp.2018.08.028

Cao J, Meng S, Chang E, Beckwith-Fickas K, Xiong L, Cole RN, Radovick S, Wondisford FE, and He L (2014) Low concentrations of metformin suppress glucose production in hepatocytes through AMP-activated protein kinase (AMPK). J Biol Chem 289:20435-20446 DOI: 10.1074/ jbc.M114.567271.

Chen EC, Khuri N, Liang X, Stecula A, Chien H-C, Yee SW, Huang Y, Sali A, and Giacomini KM (2017) Discovery of competitive and noncompetitive ligands of the organic cation transporter 1 (OCT1; SLC22A1). J Med Chem 60:2685-2696 DOI: 10.1021/acs.jmedchem.6b01317.

Chen EC, Liang X, Yee SW, Geier EG, Stocker SL, Chen L, and Giacomini KM (2015) Targeted disruption of organic cation transporter 3 attenuates the pharmacologic response to metformin. Mol Pharmacol 88:75-83 DOI: 10.1124/mol.114.096776.

Chen L, Shu Y, Liang X, Chen EC, Yee SW, Zur AA, Li S, Xu L, Keshari KR, Lin MJ, et al. (2014) OCT1 is a high-capacity thiamine transporter that regulates hepatic steatosis and is a target of metformin. Proc Natl Acad Sci USA 111:9983-9988 DOI: 10.1073/pnas.1314939111. 
Chien H-C, Zur AA, Maurer TS, Yee SW, Tolsma J, Jasper P, Scott DO, and Giacomini KM (2016) Rapid method to determine intracellular drug concentrations in cellular uptake assays: application to metformin in organic cation transporter 1-transfected human embryonic kidney 293 cells. Drug Metab Dispos 44:356-364 DOI: 10.1124/dmd.115.066647.

Deng D, Sun P, Yan C, Ke M, Jiang X, Xiong L, Ren W, Hirata K, Yamamoto M, Fan S, et al. (2015) Molecular basis of ligand recognition and transport by glucose transporters. Nature 526: 391-396 DOI: 10.1038/nature14655.

Drozdzik M, Busch D, Lapczuk J, Müller J, Ostrowski M, Kurzawski M, and Oswald S (2019) Protein abundance of clinically relevant drug transporters in the human liver and intestine: a comparative analysis in paired tissue specimens. Clin Pharmacol Ther 105:1204-1212 DOI: $10.1002 / \mathrm{cpt} .1301$

Dujic T, Zhou K, Yee SW, van Leeuwen N, de Keyser CE, Javorský M, Goswami S, Zaharenko L, Hougaard Christensen MM, Out M, et al. (2017) Variants in pharmacokinetic transporters and glycemic response to metformin: a metgen meta-analysis. Clin Pharmacol Ther 101:763-772 DOI: $10.1002 /$ cpt.567.

El-Mir MY, Nogueira V, Fontaine E, Avéret N, Rigoulet M, and Leverve X (2000) Dimethylbiguanide inhibits cell respiration via an indirect effect targeted on the respiratory chain complex I. J Biol Chem 275:223-228 DOI: 10.1074/jbc.275.1.223

Eswar N, Webb B, Marti-Renom MA, Madhusudhan MS, Eramian D, Shen M-Y, Pieper U, and Sali A (2006) Comparative protein structure modeling using modeller. Curr Protoc Bioinformatics Chapter 5:Unit-5.6 DOI: 10.1002/0471250953.bi0506s15.

Foretz M, Hébrard S, Leclerc J, Zarrinpashneh E, Soty M, Mithieux G, Sakamoto K, Andreelli F, and Viollet B (2010) Metformin inhibits hepatic gluconeogenesis in mice independently of the LKB1/AMPK pathway via a decrease in hepatic energy state. J Clin Invest 120:2355-2369 DOI: 10.1172/JCI40671.

Gorboulev V, Rehman S, Albert CM, Roth U, Meyer MJ, Tzvetkov MV, Mueller TD, and Koepsell H (2018) Assay conditions influence affinities of rat organic cation transporter 1: analysis of mutagenesis in the modeled outward-facing cleft by measuring effects of substrates and inhibitors on initial uptake. Mol Pharmacol 93:402-415 DOI: 10.1124/mol.117.110767.

Gorboulev V, Ulzheimer JC, Akhoundova A, Ulzheimer-Teuber I, Karbach U, Quester S, Baumann C, Lang F, Busch AE, and Koepsell H (1997) Cloning and characterization of two human polyspecific organic cation transporters. DNA Cell Biol 16:871-881 DOI: 10.1089/dna.1997.16.871.

Gormsen LC, Sundelin EI, Jensen JB, Vendelbo MH, Jakobsen S, Munk OL, Hougaard Christensen MM, Brøsen K, Frøkiær J, and Jessen N (2016) In vivo imaging of human 11Cmetformin in peripheral organs: dosimetry, biodistribution, and kinetic analyses. J Nucl Med $\mathbf{5 7}$ 1920-1926 DOI: 10.2967/jnumed.116.177774.

Green RM, Lo K, Sterritt C, and Beier DR (1999) Cloning and functional expression of a mouse liver organic cation transporter. Hepatology 29:1556-1562 DOI: 10.1002/hep.510290530.

Guo Y, Chu X, Parrott NJ, Brouwer KLR, Hsu V, Nagar S, Matsson P, Sharma P, Snoeys J, Sugiyama Y, et al.; International Transporter Consortium (2018) Advancing predictions of tissue and intracellular drug concentrations using in vitro, imaging and physiologically based phar macokinetic modeling approaches. Clin Pharmacol Ther 104:865-889 DOI: 10.1002/cpt.1183.

Henderson PJ and Maiden MC (1990) Homologous sugar transport proteins in Escherichia coli and their relatives in both prokaryotes and eukaryotes. Philos Trans R Soc Lond B Biol Sci 326: 391-410 DOI: 10.1098/rstb.1990.0020.

Horton RM, Hunt HD, Ho SN, Pullen JK, and Pease LR (1989) Engineering hybrid genes without the use of restriction enzymes: gene splicing by overlap extension. Gene 77:61-68 DOI: $10.1016 / 0378-1119(89) 90359-4$

Jensen O, Matthaei J, Blome F, Schwab M, Tzvetkov MV, and Brockmöller J (2020) Variability and heritability of thiamine pharmacokinetics with focus on OCT1 effects on membrane transport and pharmacokinetics in humans. Clin Pharmacol Ther 107:628-638 DOI: 10.1002/cpt.1666.

Kerb R, Brinkmann U, Chatskaia N, Gorbunov D, Gorboulev V, Mornhinweg E, Keil A Eichelbaum M, and Koepsell H (2002) Identification of genetic variations of the human organic cation transporter hOCT1 and their functional consequences. Pharmacogenetics 12:591-595 DOI: 10.1097/00008571-200211000-00002.

Lee N, Hebert MF, Wagner DJ, Easterling TR, Liang CJ, Rice K, and Wang J (2018) Organic cation transporter 3 facilitates fetal exposure to metformin during pregnancy. Mol Pharmacol 94 1125-1131 DOI: 10.1124/mol.118.112482.

Liang X, Yee SW, Chien H-C, Chen EC, Luo Q, Zou L, Piao M, Mifune A, Chen L, Calvert ME et al. (2018) Organic cation transporter 1 (OCT1) modulates multiple cardiometabolic traits through effects on hepatic thiamine content. PLoS Biol 16:e2002907 DOI: 10.1371/ journal.pbio.2002907.

Lindorff-Larsen K, Piana S, Palmo K, Maragakis P, Klepeis JL, Dror RO, and Shaw DE (2010) Improved side-chain torsion potentials for the Amber ff99SB protein force field. Proteins $\mathbf{7 8}$ 1950-1958 DOI: 10.1002/prot.22711.

Liu J, Zheng Q, Deng Y, Cheng C-S, Kallenbach NR, and Lu M (2006) A seven-helix coiled coil. Proc Natl Acad Sci USA 103:15457-15462 DOI: 10.1073/pnas.0604871103.

Lobley A, Sadowski MI, and Jones DT (2009) pGenTHREADER and pDomTHREADER: new methods for improved protein fold recognition and superfamily discrimination. Bioinformatic 25:1761-1767 DOI: 10.1093/bioinformatics/btp302.

Madeira F, Park YM, Lee J, Buso N, Gur T, Madhusoodanan N, Basutkar P, Tivey ARN, Potter SC, Finn RD, et al. (2019) The EMBL-EBI search and sequence analysis tools APIs in 2019 Nucleic Acids Res 47:W636-W641 DOI: 10.1093/nar/gkz268.

Morse BL, Kolur A, Hudson LR, Hogan AT, Chen LH, Brackman RM, Sawada GA, Fallon JK, Smith PC, and Hillgren KM (2020) Pharmacokinetics of organic cation transporter 1 (OCT1) substrates in Oct1/2 knockout mice and species difference in hepatic OCT1-mediated uptake. Drug Metab Dispos 48:93-105 DOI: 10.1124/dmd.119.088781.

Ngan C-H, Hall DR, Zerbe B, Grove LE, Kozakov D, and Vajda S (2012) FTSite: high accuracy detection of ligand binding sites on unbound protein structures. Bioinformatics 28:286-287 DOI 10.1093/bioinformatics/btr651.

Nies AT, Koepsell H, Winter S, Burk O, Klein K, Kerb R, Zanger UM, Keppler D, Schwab M, and Schaeffeler E (2009) Expression of organic cation transporters OCT1 (SLC22A1) and OCT3 (SLC22A3) is affected by genetic factors and cholestasis in human liver. Hepatology 50: 1227-1240 DOI: 10.1002/hep.23103.

Pao SS, Paulsen IT, and Saier MH Jr (1998) Major facilitator superfamily. Microbiol Mol Biol Rev 62: $1-34$.
Pei J, Tang M, and Grishin NV (2008) PROMALS3D web server for accurate multiple protein sequence and structure alignments. Nucleic Acids Res 36:W30-W34 DOI: 10.1093/nar/gkn322. Pentikäinen PJ, Neuvonen PJ, and Penttilä A (1979) Pharmacokinetics of metformin after intravenous and oral administration to man. Eur J Clin Pharmacol 16:195-202 DOI: 10.1007/bf00562061.

Quistgaard EM, Löw C, Guettou F, and Nordlund P (2016) Understanding transport by the major facilitator superfamily (MFS): structures pave the way. Nat Rev Mol Cell Biol 17:123-132 DOI: $10.1038 / \mathrm{nrm} .2015 .25$

Rena G, Hardie DG, and Pearson ER (2017) The mechanisms of action of metformin. Diabetologia 60:1577-1585 DOI: $10.1007 / \mathrm{s} 00125-017-4342-\mathrm{z}$.

Rogers AB and Dintzis RZ (2018) Hepatobiliary system, in Comparative Anatomy and Histology: A Mouse, Rat, and Human Atlas, 2nd ed (Treuting PM, Dintzis SM, and Montine KS eds) pp 229-239, Academic Press, London.

Rueden CT, Schindelin J, Hiner MC, DeZonia BE, Walter AE, Arena ET, and Eliceiri KW (2017) ImageJ2: ImageJ for the next generation of scientific image data. BMC Bioinformatics 18:529 DOI: $10.1186 / \mathrm{s} 12859-017-1934-z$.

Schindelin J, Arganda-Carreras I, Frise E, Kaynig V, Longair M, Pietzsch T, Preibisch S, Rueden C, Saalfeld S, Schmid B, et al. (2012) Fiji: an open-source platform for biological-image analysis. Nat Methods 9:676-682 DOI: 10.1038/nmeth.2019.

Schmitt A, Mössner R, Gossmann A, Fischer IG, Gorboulev V, Murphy DL, Koepsell H, and Lesch KP (2003) Organic cation transporter capable of transporting serotonin is up-regulated in serotonin transporter-deficient mice. J Neurosci Res 71:701-709 DOI: 10.1002/jnr.10521.

Seitz T, Stalmann R, Dalila N, Chen J, Pojar S, Dos Santos Pereira JN, Krätzner R, Brockmöller J, and Tzvetkov MV (2015) Global genetic analyses reveal strong inter-ethnic variability in the loss of activity of the organic cation transporter OCT1. Genome Med 7:56 DOI: 10.1186/s13073-015-0172-0.

Shitara Y, Maeda K, Ikejiri K, Yoshida K, Horie T, and Sugiyama Y (2013) Clinical significance of organic anion transporting polypeptides (OATPs) in drug disposition: their roles in hepatic clearance and intestinal absorption. Biopharm Drug Dispos 34:45-78 DOI: 10.1002/bdd.1823. Shu Y, Leabman MK, Feng B, Mangravite LM, Huang CC, Stryke D, Kawamoto M, Johns SJ, DeYoung J, Carlson E, et al.; Pharmacogenetics Of Membrane Transporters Investigators (2003) Evolutionary conservation predicts function of variants of the human organic cation transporter, OCT1. Proc Natl Acad Sci USA 100:5902-5907 DOI: 10.1073/pnas.0730858100.

Shu Y, Sheardown SA, Brown C, Owen RP, Zhang S, Castro RA, Ianculescu AG, Yue L, Lo JC, Burchard EG, et al. (2007) Effect of genetic variation in the organic cation transporter 1 (OCT1) on metformin action. $J$ Clin Invest 117:1422-1431 DOI: $10.1172 / \mathrm{JCI} 30558$.

Smith PK, Krohn RI, Hermanson GT, Mallia AK, Gartner FH, Provenzano MD, Fujimoto EK, Goeke NM, Olson BJ, and Klenk DC (1985) Measurement of protein using bicinchoninic acid. Anal Biochem 150:76-85 DOI: 10.1016/0003-2697(85)90442-7.

Sohlenius-Sternbeck A-K (2006) Determination of the hepatocellularity number for human, dog, rabbit, rat and mouse livers from protein concentration measurements. Toxicol In Vitro $\mathbf{2 0}$ 1582-1586 DOI: 10.1016/j.tiv.2006.06.003

Sundelin E, Gormsen LC, Jensen JB, Vendelbo MH, Jakobsen S, Munk OL, Christensen M, Brøsen K, Frøkiaer J, and Jessen N (2017) Genetic polymorphisms in organic cation transporter 1 attenuates hepatic metformin exposure in humans. Clin Pharmacol Ther 102:841-848 DOI: $10.1002 / \mathrm{cpt} .701$

Tucker GT, Casey C, Phillips PJ, Connor H, Ward JD, and Woods HF (1981) Metformin kinetics in healthy subjects and in patients with diabetes mellitus. Br J Clin Pharmacol 12:235-246 DOI: 10.1111/j.1365-2125.1981.tb01206.x.

Tzvetkov MV, Saadatmand AR, Bokelmann K, Meineke I, Kaiser R, and Brockmöller J (2012) Effects of OCT1 polymorphisms on the cellular uptake, plasma concentrations and efficacy of the 5-HT(3) antagonists tropisetron and ondansetron. Pharmacogenomics J 12:22-29 DOI $10.1038 /$ tpj.2010.75

Umehara K-I, Iwatsubo T, Noguchi K, and Kamimura H (2007) Functional involvement of organic cation transporter1 (OCT1/Oct1) in the hepatic uptake of organic cations in humans and rats. Xenobiotica 37:818-831 DOI: 10.1080/00498250701546012.

Wang D-S, Jonker JW, Kato Y, Kusuhara H, Schinkel AH, and Sugiyama Y (2002) Involvement of organic cation transporter 1 in hepatic and intestinal distribution of metformin. J Pharmacol Exp Ther 302:510-515 DOI: 10.1124/jpet.102.034140.

Wang D-S, Kusuhara H, Kato Y, Jonker JW, Schinkel AH, and Sugiyama Y (2003) Involvement of organic cation transporter 1 in the lactic acidosis caused by metformin. Mol Pharmacol 63: 844-848 DOI: $10.1124 / \mathrm{mol} .63 .4 .844$.

Wilcock C and Bailey CJ (1994) Accumulation of metformin by tissues of the normal and diabetic mouse. Xenobiotica 24:49-57 DOI: 10.3109/00498259409043220.

Yabe Y, Galetin A, and Houston JB (2011) Kinetic characterization of rat hepatic uptake of 16 actively transported drugs. Drug Metab Dispos 39:1808-1814 DOI: 10.1124/dmd.111.040477.

Zhang L, Dresser MJ, Gray AT, Yost SC, Terashita S, and Giacomini KM (1997) Cloning and functional expression of a human liver organic cation transporter. Mol Pharmacol 51:913-921 DOI: $10.1124 / \mathrm{mol} .51 .6 .913$.

Zhou G, Myers R, Li Y, Chen Y, Shen X, Fenyk-Melody J, Wu M, Ventre J, Doebber T, Fujii N et al. (2001) Role of AMP-activated protein kinase in mechanism of metformin action. J Clin Invest 108:1167-1174 DOI: $10.1172 / \mathrm{JCI} 13505$.

Zhou K, Donnelly LA, Kimber CH, Donnan PT, Doney ASF, Leese G, Hattersley AT, McCarthy MI, Morris AD, Palmer CNA, et al. (2009) Reduced-function SLC22A1 polymorphisms encoding organic cation transporter 1 and glycemic response to metformin: a GoDARTS study. Diabetes 58:1434-1439 DOI: 10.2337/db08-0896.

Zhu BY, Zhou NE, Kay CM, and Hodges RS (1993) Packing and hydrophobicity effects on protein folding and stability: effects of beta-branched amino acids, valine and isoleucine, on the formation and stability of two-stranded alpha-helical coiled coils/leucine zippers. Protein Sci 2 : 383-394 DOI: 10.1002/pro.5560020310.

Address correspondence to: Dr. Mladen V. Tzvetkov, Institute of Pharmacology, Center of Drug Absorption and Transport, University Medicine Greifswald, FelixHausdorff-Str. 3, 17489 Greifswald, Germany. E-mail: mladen.tzvetkov@med.unigreifswald.de 